\title{
Automatic priming of attentional control by relevant colors
}

\author{
Ulrich Ansorge • Stefanie I. Becker
}

Published online: 21 October 2011

(C) Psychonomic Society, Inc. 2011

\begin{abstract}
We tested whether color word cues automatically primed attentional control settings during visual search, or whether color words were used in a strategic manner for the control of attention. In Experiment 1, we used color words as cues that were informative or uninformative with respect to the target color. Regardless of the cue's informativeness, distractors similar to the color cue captured more attention. In Experiment 2, the participants either indicated their expectation about the target color or recalled the last target color, which was uncorrelated with the present target color. We observed more attentional capture by distractors that were similar to the participants' predictions and recollections, but no difference between effects of the recollected and predicted colors. In Experiment 3, we used 100\%informative word cues that were congruent with the predicted target color (e.g., the word "red" informed that the target would be red) or incongruent with the predicted target color (e.g., the word "green" informed that the target would be red) and found that informative incongruent word cues primed attention capture by a word-similar distractor. Together, the results suggest that word cues (Exps. 1 and 3) and color representations (Exp. 2) primed attention capture in an automatic manner. This indicates that color cues
\end{abstract}

U. Ansorge ( $\square)$

Fakultät für Psychologie, Universität Wien,

Liebiggasse 5,

1010 Wien, Austria

e-mail: ulrich.ansorge@univie.ac.at

\section{U. Ansorge}

Institute for Cognitive Science, Universität Osnabrück,

Osnabrück, Germany

U. Ansorge · S. I. Becker

School of Psychology, University of Queensland,

Brisbane, Australia automatically primed temporary adjustments in attention control settings.

Keywords Spatial attention - Attentional capture . Top-down control · Vision

Visuospatial attention is the mechanism by which humans can select one stimulus out of several objects that are concurrently present in the visual world to use the selected stimulus as a target for discrimination, identification, and behavior. One of the crucial questions in this domain is how humans exert top-down control over visuospatial attention (cf. Bichot, Rossi, \& Desimone, 2005; Duncan \& Humphreys, 1989). Experimentally, top-down control over attention is demonstrated by contingent capture (cf. Folk, Remington, \& Johnston, 1992). In a typical contingentcapture experiment, participants have to search for a feature-defined target stimulus - for example, a red target. In this situation, an irrelevant distractor stimulus with a color similar to the searched-for target (e.g., red) captures attention, whereas differently colored distractors (e.g., green) can mostly be ignored and capture attention much less or not at all (cf. Ansorge, Kiss, Worschech, \& Eimer, 2011; Folk \& Remington, 1998). According to the contingent-capture account, selective capture by targetsimilar distractors is due to the distractor matching a topdown target template that participants strategically set up in search for the target (cf. Folk et al., 1992).

The contingent-capture effect has been demonstrated in a multitude of studies. However, it has been argued that capture by target-similar distractors does not necessarily reflect top-down control of attention, but could instead reflect automatic selection of the color corresponding to the target color on the previous trial (e.g., Theeuwes, Reimann, \& Mortier, 2006). In experiments in 
which the target color varied randomly (e.g., between red and green), it has been found that selection of the target on a given trial can automatically prime attention shifts to that color on subsequent trial(s) (e.g., Maljkovic \& Nakayama, 1994). In experiments on the contingent-capture effect, the target color typically never changes, so it is possible that capture by target-similar distractors is not driven by a topdown target template, but by automatic intertrial carryover effects that prime selection of the color that the target had on the previous trial(s).

Studies examining the contributions of intertrial priming and top-down target templates on capture, however, have not supported the automatic-priming explanation of the contingent-capture effect: In studies in which the target color varied randomly between red and green, it was found that a red or green distractor can capture attention even when the previous target had a different color (Ansorge \& Heumann, 2003, 2004; Ansorge \& Horstmann, 2007). At most, the color of the target on the previous trial modulated capture such that intertrial-primed color distractors captured attention more strongly (Folk \& Remington, 2008), but priming was not decisive for capture. In addition, when one is searching for green and red targets, a red distractor captures attention even when the preceding and the current target are green (Ansorge \& Horstmann, 2007). This result suggests that the likely origin of the contingent-capture effect is the match between the target search template and the distractor color, rather than a correspondence between the color of the distractor and that of the subsequent target. Also, participants can evidently strategically select items in accordance with the instructions to search for two particular colors and/or to ignore a third color. This is reflected in the finding that a different color distractor with a third, additional color fails to capture attention even when the participants successfully search for two colors (cf. Ansorge \& Heumann, 2004; but see Folk \& Anderson, 2010).

A large part of the research concerning the concept of contingent capture has focused on average capture effects (cf. Folk \& Remington, 1998; Folk et al., 1992; for a review, see Burnham, 2007). However, less is known about the exact way in which the attentional control settings are temporarily adjusted to changing search requirements. Clearly, the flexible choice of a particular color (or color difference; cf. Becker, Folk, \& Remington, 2010) for the attentional control settings entails that humans can strategically and voluntarily select the feature templates used in their attentional control settings. However, recent research has suggested that the participants' strategic control over the temporary adjustments of the control settings is limited: Once a particular feature, such as a specific color, is associated with the target, selection is apparently primed or biased toward selecting same-color items on subsequent trials in an automatic fashion (e.g., Maljkovic \& Nakayama,
1994). For example, if observers search for a target that is randomly either green or red and is presented among distractors of the opposite color, the target is selected faster when the colors of the target and distractors repeat across consecutive trials than when the colors switch (e.g., Becker, 2008a, b; Belopolsky, Schreij, \& Theeuwes, 2010). Similarly, presenting a different-feature target (e.g., a shape target) in a particular color can prime attention shifts toward same-colored items on subsequent trials, even when color is irrelevant for the task (Maljkovic \& Nakayama, 1994). Maljkovic and Nakayama showed that priming modulated attention shifts to the target even when the colors of the target and distractors changed in a regular, foreseeable fashion (e.g., in sequences of $\mathrm{ABABAB}, .$. . , AABBAABB, ... . ; see also Becker, 2008b; Hillstrom, 2000) and when participants were required to name the upcoming target's color prior to the trial. These findings led Maljkovic and Nakayama to conclude that priming is due to a top-down-impenetrable memory system for attentional deployments (e.g., Kristjánsson \& Nakayama, 2003; Nakayama, Maljkovic, \& Kristjánsson, 2004) that modulates attention in a rapid, automatic or "stimulus-driven" way (but see Geyer \& Müller, 2009; Hillstrom, 2000). Also, even theories admitting that capture and priming are contingent on task relevance do not deny the automatic nature of priming of attentional control settings ("contingent automaticity"; Bargh, 1992). Priming effects may be contingent on using a nominally irrelevant feature and/or deploying attention to such a feature; however, once an item is attended, selection of same-feature items on the next trial would be facilitated automatically and without the need of actively changing top-down control settings on a trialby-trial basis (e.g., the "contingent-priming hypothesis"; Becker, 2007; Olivers \& Humphreys, 2003).

Another line of evidence for this concerns the automatic temporary priming of attentional control settings by features outside of the range of search-relevant features but contained in the working memory (WM) of participants (cf. Olivers, 2009; Olivers, Meijer, \& Theeuwes, 2006; Soto, Heinke, Humphreys, \& Blanco, 2005; but see, e.g., Woodman \& Luck, 2007). To date, there is some evidence that WM content can automatically prime attention shifts, regardless of the usefulness of that content for the task of finding the target (cf. Olivers, 2009). In a typical WM experiment on attention, at the outset of a trial participants have to encode a stimulus together with its features (e.g., its red color) and have to keep the stimulus in memory for later recall. However, during the time interval between encoding and recall, the participants have to search for a relevant target of another feature (e.g., a green target) among irrelevant distractors. Yet attention capture is seemingly driven in an automatic and nonstrategic way by whatever is held in WM: A distractor with a feature similar to the 
content of WM has the potential to attract attention during visual search (Olivers, 2009). Thus, it seems that once a feature is relevant because, for instance, it has to be kept in mind for later recall, the WM content intrudes into the attentional control settings and guides attention toward similar items, even when this produces visual search costs. In general agreement with this possibility of automatic (or conditionally automatic) priming, even the meaning of a word or an object that is presented below the level of participants' aware perception is processed and primes processing of a particular dimension of a subsequent relevant target stimulus (Kiefer, 2002; Martens, Ansorge, \& Kiefer, 2011).

By contrast, other studies have suggested that participants strategically control even the temporary adjustments of their control settings. Firstly, Wolfe, Butcher, Lee, and Hyle (2003) emphasized that priming might reflect the (implicit) expectations of observers about the feature of the upcoming target rather than a completely automatic stimulus-driven process. In line with the role of participants' expectancies about the next feature, it has been shown that intertrial priming of attentional capture across subsequent trials can be boosted or attenuated by manipulating the reward that the participants receive and expect for their correct responses to a particular feature: Intertrial priming effects of attentional capture were stronger for targets with a higher-rewarded feature than for less-rewarded target features (Kristjánsson, Sigurjónsdóttir, \& Driver, 2010). This finding entails the possibility that the participants strategically used a particular (e.g., repeated) feature-here, the rewarded feature-for their temporary adjustments of the attentional control settings, and that strategies thus could be crucial for intertrial priming of these control settings.

Secondly, and relatedly, a number of studies have shown that priming effects can be modulated in response to a word or object cue that provided information about the upcoming target. For instance, Fecteau (2007) created ambiguous displays containing both a shape and a color singleton that could have different features (e.g., red or green) and asked participants to search for the singleton specified by a cue prior to the trial. In this condition, intertrial priming strongly depended on the task relevance of the attended feature: Search was faster when the task-relevant feature was repeated (as compared to when it was switched), but not when the task-irrelevant feature was repeated. This indicated that priming depends on top-down attentional control settings (for related results, see also Exps. 4 and 5 of Belopolsky et al., 2010). Likewise, Leonard and Egeth (2008) tested capture by irrelevant red or green distractors when the target was randomly red or green, and participants were informed with $100 \%$ certainty about the color of the upcoming target. The cue had a strong mitigating effect on attention capture by the irrelevant color distractors and on cumulative intertrial priming of color capture.
In sum, there is currently good evidence that valid precueing can reduce intertrial priming effects on attentional capture. This effect has uniformly been interpreted as showing that intertrial priming effects are to some extent top-down penetrable or are contingent on top-down attentional control settings (e.g., Fecteau, 2007; Leonard \& Egeth, 2008). However, contrary to this interpretation, it is possible that color words or colored objects at the beginning of a trial can prime attentional control settings automatically, much as a relevant feature in a preceding trial does. Here, we set out to investigate whether word cues at the beginning of the trial are strategically used or whether they modulate attentional capture in an automatic fashion.

\section{Experiment 1}

Experiment 1 tested the effects of informative (75\% valid) versus uninformative ( $50 \%$ valid) word cues on attention capture by a color distractor. Participants had to search for a single target line that was presented against the background of a colored semidisk and to report the line's orientation. On all trials, an irrelevant distractor of a different color was presented either at the location of the target (SP: same position) or away from it (DP: different position). The target and distractor could be either red or green, and the two stimuli always had opposing colors (i.e., when the target was green, the distractor was red, and vice versa; see Fig. 1 for an example). In this experiment, the contents of the attentional control settings could be tested by comparing

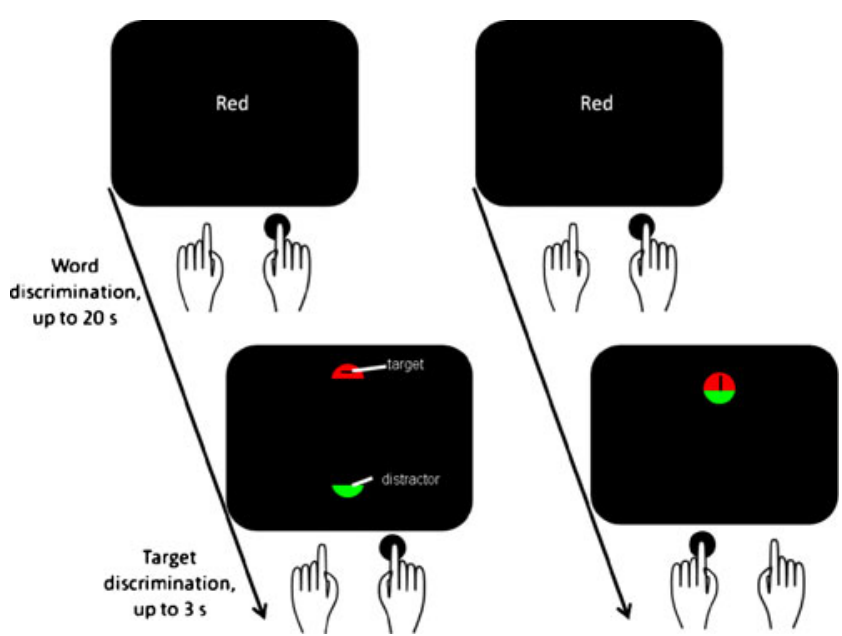

Fig. 1 Schematic examples of the sequences of events in a DP (different distractor-target position) trial (on the left) and an SP (same distractor-target position) trial (on the right) of Experiment 1, with a cue-similar target (on the left and on the right). The arrow depicts the direction of time. Also depicted are buttonpresses to confirm the identity of the cue and start target search, and to discriminate the target. (Note that the pertaining mapping of color cues to responses was also repeated at the beginning of the trial [not depicted].) Stimuli are not drawn to scale 
the amount of attention capture by an irrelevant distractor that was either similar or dissimilar to a word color cue at the beginning of a trial: When presenting the word cue "red" at the outset of a trial tunes attention more to red (than to green), search will be greatly impaired when the distractor is red and presented at a different position (DP) from the target, because word-primed attention will first select the red distractor and needs to be reallocated to the target in a time-consuming process. Search would be less impaired when the distractor is presented at the same position (SP) as the target. Finally, the red DP distractor will not impair search as strongly when it is dissimilar to the word and attention is, for example, set toward green. Thus, the contents of the attentional control settings can be assessed by comparing distractor-target position effects (DP - SP) in different cue-distractor or word-distractor similarity combinations.

Critically, we tested whether word cues affected attentional control settings strategically or in an automatic fashion by comparing word priming of attention capture when the word cue informed about the target color $(75 \%$ valid) versus when it did not inform about the target color ( $50 \%$ valid). The informativeness of the cue was manipulated between blocks, and participants were informed about the average accuracy of the word cue prior to each block. Also, in each trial, after the color word cue appeared and prior to the target search display, participants had to discriminate which word had been presented as a cue to them. In this manner, we ensured that the participants noticed and represented the current color cue. Participants then started the presentation of the target search display in a self-paced manner (by pressing a key). This was done to ensure that participants had sufficient preparation time for the strategic use of the color cue.

If words affected attentional capture via top-down control (cf. Fecteau, 2007; Leonard \& Egeth, 2008) and participants have strategic control over the temporary trialby-trial changes of their attentional control settings, we expected to find differences in the distractor's attention capture effects between the color-informative and coloruninformative blocks. Participants should use the color word for the specification of their attentional control settings in the trials of the color-informative blocks, but not in the color-uninformative blocks. Hence, where the distractor rather than the target had the cued color, distractor interference should be stronger in the colorinformative blocks than in the color-uninformative blocks. By contrast, in uninformative blocks, distractor interference should be independent of whether or not the distractor had the announced color, because the color word should not be tipping the participants' strategies to set up control settings for one or the other color.

However, if word cues can automatically prime attentional control settings, then distractors with the cued or primed color should always capture attention more strongly than distractors with a cue-dissimilar color, regardless of the informativeness of the word cue. The reason for this expectation was that the participants had to discriminate, and therefore represent, the color word cues in each trial of both blocks. Therefore, the necessary conditions for automatic priming of the attentional control settings - as these have been given in WM experiments, for examplewere fulfilled in both blocks.

To also assess intertrial priming effects, we compared the attentional effects of distractors (i.e., the DP-SP differences), depending on whether the current trial $n$ 's target-distractor color combinations were repeated or switched, as compared to the previous, $n-1$, trial. In this manner, we could test whether the word priming of attention capture overrules intertrial priming of capturefor example, because the color word is even closer in time to the distractor and target.

\section{Method}

Participants A group of 16 volunteers ( 8 female, 8 male; ages 19-28 years, mean age 22 years) received course credit to participate in this study. The participants had normal or corrected-to-normal vision.

Stimuli and procedure Visual stimuli were presented on a 15-in. color VGA monitor. Its refresh rate was $59.1 \mathrm{~Hz}$. The participants sat at a distance of $57 \mathrm{~cm}$ from the screen in a quiet, dimly lit room, with their head resting in a chinrest to ensure a constant viewing distance and a straight-ahead gaze direction. Reaction times (RTs) and response identities were registered via the " $\mathrm{X}$ " and "C" keys on a standard computer keyboard, placed directly in front of the participants. The keys were pressed with the left and right index fingers, respectively.

For the sequence of events in a trial, see Fig. 1. On each trial, a color was announced by presenting the word "green" as a cue on half of the trials and the word "red" on the other half of the trials. Together with the word, participants saw instructions about which key (left or right) had to be pressed for which cue (e.g., right key for "green"/left key for "red"). (The mappings were fixed, but the instructions were repeated nonetheless.) Participants were given ample time (up to $20 \mathrm{~s}$ ) to respond to the word cue and to initiate the next trial and were encouraged to use the color information in the preparation of the search templates if an informative cue was used. After pressing the key, the screen went blank and two semidisks, one upper-half semidisk and one lower-half semidisk (both of $0.5^{\circ}$ length and $0.25^{\circ}$ height), were presented simultaneously as the target (containing a target line) and the distractor (without a line) until a response was given or $3 \mathrm{~s}$ had elapsed. The 
disks were shown above or below screen center, with an eccentricity of $3.9^{\circ}$ to the base of the semidisks. If the target was an upper-half semidisk, the distractor was a lower-half semidisk, and if the target was a lower-half semidisk, the distractor was an upper-half semidisk. If the target was green $(\mathrm{CIE}$ color coordinates $=.261 / .561)$, the distractor was red (.640/.347), and if the target was red, the distractor was green. On half of the trials, the target and distractor were shown adjacent to one another: Either both were presented below the screen center or both were shown above the screen center. This was the SP condition. On the other half of the trials, the target was shown on the side opposite the distractor. This was the DP condition. On $25 \%$ of the trials, the target was shown above the screen center and the distractor was shown below the screen center, whereas the positions of the target and distractor were exchanged on the remaining $25 \%$ of all trials.

Only the target contained a vertical or a horizontal line $\left(0.08^{\circ}\right.$ wide, $0.44^{\circ}$ long $)$ of the color of the background (black), and participants had to find this line and report its orientation: Participants pressed the left key for one orientation and the right key for the other orientation (with the mappings of orientations to keys balanced across participants).

Design The experiment consisted of two blocked conditions that only differed with respect to the relationship between color announcement (or word cue) and target color. In the color-uninformative blocks, color announcement and target color were uncorrelated. In color-informative blocks, color announcements correctly informed about the target color on $75 \%$ of the trials and incorrectly informed about the target color on $25 \%$ of the trials.

To rule out influences of the sequence of one blocked condition on the other blocked condition, we repeated the $50 \%$ color-uninformative and the $75 \%$ color-informative blocks and presented the differently informative blocks in alternating order; half of the participants started with an informative block (with the block sequence informative-uninformative-informativeuninformative, hereafter abbreviated " $\mathrm{i}-\mathrm{u}-\mathrm{i}-\mathrm{-u}$ "), and half with an uninformative block (with the block sequence uninformative-informative-uninformative-informative, hereafter abbreviated " $\mathrm{u}-\mathrm{i}-\mathrm{u}-\mathrm{i}$ "). Every one of the four "miniblocks" included 128 trials: four repetitions of the 32 combinations of 2 target colors (green or red) $\times 2$ target shapes (upper or lower half of a semidisk) $\times 2$ line orientations (horizontal or vertical) $\times 2$ target positions (above or below screen center) $\times 2$ distractor-target positions (SP or DP). Prior to the start of each block, participants were informed about the average accuracy with which the color word announced the correct target color in a trial of the upcoming block, and participants were encouraged to use this predictive relationship (if it existed; i.e., in color-informative blocks) to guide their target localization. A brief practice phase preceded the experiment.

\section{Results}

Color word discrimination At the beginning of every trial, we manipulated the color representation of our participants by presenting a color word cue that informed or did not inform about the color of the subsequent target. In a first ANOVA, with the within-participants variables block (color-informative vs. -uninformative) and intertrial priming (color repetition [color cue same as preceding target color] vs. color switch [color cue not the same as preceding target color]), we investigated whether this manipulation had worked out as intended and whether our participants correctly discriminated the color words. This was the case. Accuracy was high in color-informative blocks (97.6\%) and in color-uninformative blocks $(97.8 \%)$ and did not differ significantly between blocks, $F<1$. Accuracy was also the same in the intertrial color-repeated and color-switch conditions $(97.6 \%$ vs. $97.7 \%), F<1$. There was also no significant block $\times$ priming interaction, $F<1$. In addition, a slightly longer color word discrimination time in the informative blocks $(M=742 \mathrm{~ms})$ as compared to the uninformative blocks $(M=715 \mathrm{~ms}), t(15)=1.81, p<.05$ (one-sided), supported the assumption that only in the informative blocks did the participants take additional time to prepare a search template in accordance with the color words.

Target search See Fig. 2 and Table 1 for the target search results. Out of all trials, $4.6 \%$ were eliminated from analyses because of RTs that differed from the individual mean correct RT by more than two standard deviations. We ran a repeated measures ANOVA restricted to the correct target responses of trials with correct color word discriminations. The ANOVA was based on the within-participants variables informativeness (color-informative vs. coloruninformative block), distractor-target positions (SP vs. DP), and word-target similarity (word-target color similar/ word-distractor dissimilar vs. word-target color dissimilar/ word-distractor similar). In RTs, there was first of all a strong effect of distractor position; RTs were much faster on SP trials $(M=669 \mathrm{~ms})$ than on DP trials $(M=722 \mathrm{~ms}), F(1$, $15)=84.88, p<.01$. Importantly, this spatially specific capture effect (RT in DP trials minus RT in SP trials) of the distractors was significantly larger for distractors that were similar (and targets that were dissimilar) to the color words (capture effect $=61 \mathrm{~ms}$ ) than for distractors that were dissimilar (and targets that were similar) to the color words (capture effect $=45 \mathrm{~ms}$ ): word-target similarity $\times$ dis- 


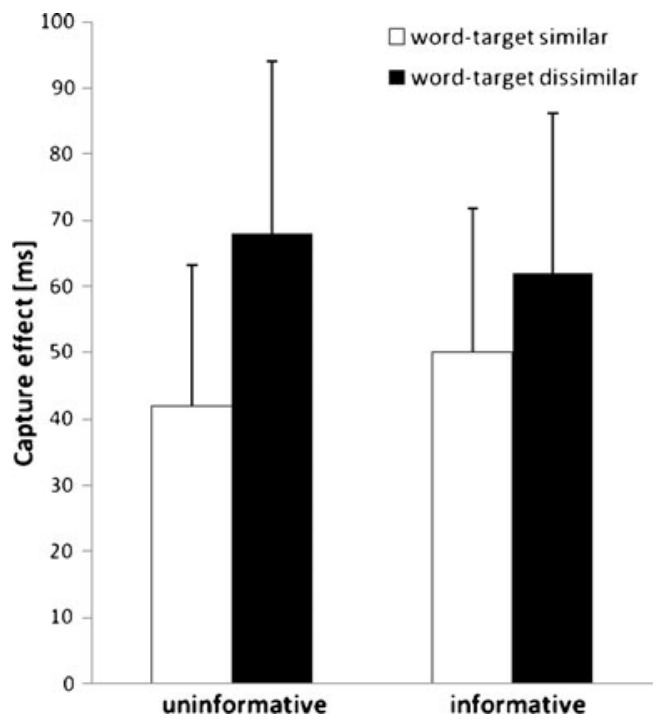

Fig. 2 Experiment 1's mean capture effects (calculated as RT in the DP conditions minus RT in the SP conditions) plus their corresponding standard errors (line bars), separately for the uninformative and informative blocks and for the two different target colors (word-target similar [word-distractor dissimilar] or word-target dissimilar [word-distractor similar]; white and black histograms)

tractor-target position interaction, $F(1,15)=9.39, p<.01$. However, follow-up analyses revealed a reliable spatial capture effect by cue-similar as well as -dissimilar distractors [both $t \mathrm{~s}(15)>7.90$, both $p \mathrm{~s}<.01$ ].

Critically for our question, color words primed attention shifts to the distractor in an automatic manner: Whether the words were informative or uninformative with respect to the color of the target (and the distractor) neither had an effect on the capture effect (informativeness $\times$ distractortarget position interaction, $F<1$ ), nor modulated the effects of distractors that were similar versus dissimilar to the cued color (informativeness $\times$ distractor-target position $\times$ wordtarget similarity interaction, $F<1$ ).

In addition, we found a significant main effect of wordtarget similarity, $F(1,15)=24.98, p<.01$, and a significant informativeness $\times$ word-target similarity interaction, $F(1$,
$15)=10.73, p<.01$. Responses were faster when the color word primed the target $(M=678 \mathrm{~ms})$ than when the color word primed the distractor $(M=712 \mathrm{~ms})$. The two-way interaction reflected that this spatially unselective wordtarget priming effect was stronger with uninformative [priming effect (unprimed RT minus primed RT) $=51 \mathrm{~ms}, t(15)=$ $5.16, p<.01$ ] than with informative [priming effect $=18 \mathrm{~ms}$, $t(15)=2.63, p<.05]$ cues. (The main effect of informativeness was not significant, $F<1$.)

A similar ANOVA on error rates (ERs) showed a significant interaction between word-target similarity and distractor-target position, $F(1,15)=6.06, p<.05$. With distractors dissimilar to the words (and targets similar to the words), the spatial capture effect of the distractors [ER(DP) $\operatorname{ER}(\mathrm{SP})]$ was stronger $[2.3 \%, t(15)=2.61, p<.05]$ than with distractors similar to the words (and targets dissimilar to the words; $0.5 \%, t<1$ ). This means that at least part of the stronger RT capture effect of the word-resembling distractors could have also reflected a tendency to trade accuracy for faster responses. Crucially, again, the two interactions that included the variables distractor-target position and informativeness were far from significant, both $F_{\mathrm{S}}<1$. Neither of the main effects, all $F_{\mathrm{S}}<3.10$, all $p \mathrm{~s}>.10$, nor the remaining interactions, all $F \mathrm{~s}<1$, were significant.

Additional analyses A first additional ANOVA included the between-participants variable block order $(\mathrm{i}-\mathrm{u}-\mathrm{i}-\mathrm{u}$ vs. $\mathrm{u}-\mathrm{i}-\mathrm{u}-\mathrm{i})$. This variable created no significant main effect, $F(1,14)=1.17, p=.29$, and it did not significantly interact with any other variable of interest, all $F \mathrm{~s}<2.70$, all $p \mathrm{~s}>.12$.

Secondly, the error rates indicated that a speed-accuracy trade-off could have accounted for the interaction between word-target similarity and distractor-target position. To confirm that the words indeed primed capture by wordsimilar distractors, we therefore repeated our initial analysis with individual RTs weighted by the individual ratios of correct as compared to incorrect answers. This ANOVA replicated all major findings reported above [distractor-target position, $F(1,15)=23.03, p<.01$; word-target similarity, $F$
Table 1 Mean reaction times (RTs, in milliseconds) and error rates (ERs), plus standard deviations in parentheses, as a function of word-target similarity (distractor color dissimilar vs. similar to word), informativeness $(75 \%$ vs. $50 \%)$, and targetdistractor position relation (same position [SP] vs. different position [DP]) in Experiment 1

\begin{tabular}{lllll}
\hline Distractor Color & Informativeness & Position & RT (SD) & ER (SD) \\
\hline Dissimilar to word & $75 \%$ & SP & $661(81)$ & $3.9(3.9)$ \\
& & DP & $709(93)$ & $5.9(6.1)$ \\
Similar to word & $50 \%$ & SP & $650(78)$ & $4.1(3.7)$ \\
& \multirow{2}{*}{$75 \%$} & DP & $691(90)$ & $6.7(5.2)$ \\
& $50 \%$ & SP & $671(86)$ & $5.2(4.0)$ \\
& & DP & $734(107)$ & $5.5(3.6)$ \\
& SP & $692(91)$ & $4.6(4.5)$ \\
& & DP & $752(117)$ & $5.1(5.4)$ \\
\hline
\end{tabular}


Table 2 Mean RTs (plus standard deviations in parentheses) as a function of intertrial target color priming (yes [color of target repeats across trials] vs. no [color of target switches across trials]), informativeness ( $75 \%$ vs. $50 \%)$ and target-distractor position relation (same position [SP] vs. different position [DP]) in Experiment 1

\begin{tabular}{llll}
\hline Intertrial Priming & Informativeness & Position & RT (SD) \\
\hline Yes & $75 \%$ & SP & $660(85)$ \\
& & DP & $721(116)$ \\
& $50 \%$ & SP & $649(80)$ \\
No & & DP & $707(97)$ \\
& $75 \%$ & SP & $676(83)$ \\
& & DP & $720(93)$ \\
& $50 \%$ & SP & $669(84)$ \\
& & DP & $704(98)$ \\
\hline
\end{tabular}

$(1,15)=13.65, p<.01$; word-target similarity $\times$ targetdistractor position, $F(1,15)=19.66, p<.01$; informativeness $\times$ word-target similarity, $F(1,15)=9.54, p<.01$; all other $F \mathbf{s}<1]$.

Thirdly, at least in one study, it was reported that an informative color word cue (e.g., the word "green"), but not an uninformative neutral word cue (e.g., the word "either"), eliminated cumulative intertrial priming of attentional capture, and this was taken as an argument for the strategic use of the color word cue on the distractors' capture effects (Leonard \& Egeth, 2008). To test whether the informative and uninformative color word cues of the present study differed in their power to overcome intertrial priming of capture, we ran a second, complementary ANOVA of the correct RTs, with the variables distractor-target position, informativeness, and intertrial priming (target color repetition $[n-1=n]$ vs. target color switch $[n-1 \neq n])$. On the basis of Leonard and Egeth's (2008) findings, we expected more capture by the distractors in target color switch than target color repetition trials after uninformative but not after informative color word cues. However, this was not observed (see Table 2). First, if anything, DP distractors captured more attention after repeated target colors (capture effect $=59 \mathrm{~ms}$ ) than after switched target colors (capture effect $=40 \mathrm{~ms}): F(1,15)=6.66, p>.05$, for the interaction between distractor-target position and intertrial priming. Secondly, this modulating influence of intertrial priming was the same for informative and uninformative color words: $F<1$ for the three-way interaction of all variables.

\section{Discussion}

In line with automatic priming of attentional control settings, we found that color words at the beginning of a trial biased search toward the announced color: Search was significantly slower and capture by the distractor greater when the word cued the distractor color rather than the target color, indicating that the words primed attention toward selecting word-similar items. Importantly, none of these effects was significantly modulated by the informativeness of the word cue. This indicated that word cues biased attention in an automatic fashion and independently of the observer's strategies to use the color information in search. Thus, the results of Experiment 1 indicated that the task to attend to and distinguish between different color words was sufficient to bias attention toward that color, without the additional requirement that the cue be of help in locating the target. With this finding, the present results are in line with those of WM studies showing automatic priming of attention by WM content, and they call into question the view that the effects of informative word cues on attentional capture are necessarily top-down controlled and dependent on the observer's strategies.

However, two caveats seem to be in order. Firstly, it should be noted that Experiment 1 did not show any evidence for automatic intertrial priming of attentional capture, but rather a nonsignificant trend in the opposite direction. The failure to observe intertrial priming of capture might have been due to the additional word discrimination task: It is possible that this task eliminated the effect of the previous trial, either by elongating the intertrial intervals or by overwriting the information from the previous trial. In any case, it is important to note that the results demonstrate automatic biasing of attention by word cues, but not an automatic modulation of intertrial priming effects by word cues; hence, it is still possible that the effects of word cues on intertrial priming effects are indeed strategic and not automatic, as has been claimed in previous studies (e.g., Leonard \& Egeth, 2008).

Secondly, we do not know exactly why spatially unselective within-trial color priming of RTs was stronger in the color-uninformative blocks than in the color-informative blocks. Stronger word similarity or priming effects in the color-uninformative blocks might have been due to the fact that the participants' attempts to strategically use the color words increased variance, and thus weakened the word priming effect in the colorinformative blocks. Whereas this explanation is highly speculative and requires further investigation, it is clear that word cues can bias attention in a purely automatic fashion. Note that the observed effects cannot be explained by later processes concerned, for example, with decisions or response selection: Such processes were not involved in the manipulation of colors and color words, as participants were required to respond to a different feature (orientation of a line) that was varied independently of the color.

In summary, the results confirmed the view that automatic priming of capture could be responsible for the temporary 
dominance of features in attentional control settings. This finding casts doubts on the interpretation of word effects on attentional capture as being strategically mediated (cf. Belopolsky et al., 2010; Fecteau, 2007; Leonard \& Egeth, 2008). We would argue that as long as the utility of a feature prediction by a word or a stimulus is not systematically varied, it is impossible to determine the extents to which feature priming of the attentional control settings indeed reflects strategic control and to which it reflects automatic feature priming of the attentional control settings.

However, Experiment 1 cannot address the important question of what the participants expected at the beginning of a trial. So far, we have assumed that the participants' expectancies reflected the word's information and that participants expected the $75 \%$ correctly announced target color (i.e., in the informative blocks) and searched for this color to find the next target, which was not necessarily the case when they could not benefit from the word cues (i.e., in the uninformative blocks). In fact, the best strategy of the participants would have been to expect the announced color target and to search for it in every trial of the colorinformative blocks, but to ignore the color words in all of the trials of the uninformative blocks.

However, it is possible that the participants did not even register the tiny advantages in search time that were provided by the informative words. In general, the subjective expected utility can be much lower than the objective advantage that is created in a particular situation (Savage, 1954). Likewise, the opposite is possible, and participants might not have bothered with changing their expectations between blocks, but might have strategically used the words in all blocks because of their average across-block utility for predicting the target colors with an above-chance probability. Finally, if the participants became aware of the benefits that the word cues created, it is possible that the probabilities of correct target color announcements influenced our participants' expectancies of the target color in a more complex manner. According to the probability-matching principle, participants tend to shape their expectations according to the probabilities of each of the different predictions (e.g., Gaissmaier \& Schooler, 2008). Thus, our participants might have expected the predicted target color on only some of the trials of the color-informative blocks, and they might have expected the unpredicted targets on a fraction of $25 \%$ of the trials, too. If, however, the participants' expectancies about the upcoming target colors had been different from the color word cues on a portion of the trials, we would have underestimated the participants' strategic control over the temporary changes of their attentional control settings in Experiment 1.

Ultimately, to understand whether or not a priming effect of an attentional control setting reflects strategic or automatic processing, we need to know for each trial what target color the participants expect. Therefore, we conducted Experiment 2, in which we left out the color word cues and asked participants for their own, freely chosen predictions of the color of the upcoming target.

\section{Experiment 2}

In Experiment 2, we asked our participants to either predict the upcoming target color (in the prospective blocks) or to recall the preceding target color (in the retrospective blocks). The prospective blocks thus directly measured the participants' expectations, avoiding the pitfalls of designs in which the participants' expectations have to be inferred from assumptions of rationality.

If the participants' expectations modulate attention, we would expect more interference when the distractor was similar to the expected feature than when the distractor was dissimilar (and the target similar) to the expected feature: As in Experiment 1, interference from distractors when they were presented away from as compared to at the target position should be higher with distractors that were similar than with distractors that were dissimilar to the expected colors.

Also if participants have strategic control over the temporary changes of their attentional control settings, their recollections about the previous target color (required in the retrospective blocks) should not affect attention. This holds because, in the retrospective blocks, the color of the target in the preceding trial was uncorrelated with the color of the target in the current trial, and participants were informed of this prior to the task. Hence, if changes in the attentional control settings depend on intentions and strategies, then only the predicted colors (in the prospective blocks) should be used for the specification of attentional control settings, and these should exert stronger effects on attention than do recollected colors (in the retrospective blocks).

However, again, if represented features automatically facilitate the temporary dominance of one feature over the other in attentional control settings, stimuli with a predicted color as well as stimuli with a recollected color should lead to more capture than do stimuli with a nonpredicted or a nonrecollected color.

Method

Participants A group of 16 volunteers (10 female, 6 male; ages 22-28 years, mean age 24 years) received course credit to participate in this study. The participants had normal or corrected-to-normal vision. 
Stimuli, procedure, and design These were exactly as in Experiment 1, with two exceptions. First, at the beginning of each trial, no color word was presented. Second, the task of the participants was to predict the color of the upcoming target in half of the blocked conditions, and it was to recollect the color of the preceding target in the other half of the blocked conditions. Participants indicated their choices with a keypress, pressing one key for one predicted or recollected color and the other key for the alternative predicted or recollected color. Again, the blocks alternated in a regular fashion, with half of the participants starting with the prospective blocks (block order: prospectiveretrospective-prospective-retrospective, abbreviated " $\mathrm{p}-\mathrm{r}-\mathrm{p}-$ r") and half of the participants starting with the retrospective blocks (block order: retrospective-prospective-retrospectiveprospective, abbreviated "r-p-r-p").

Analysis ANOVAs of the correct individual median RTs to the targets and of the ERs for targets were run twice, once restricted to trials with correct color recollections in the retrospective blocks (see the Results section below), and once with all recollections in the retrospective blocks (see Table 3). These second ANOVAs were run for the sake of a fair comparison of the retrospective and prospective blocks; in the prospective blocks, all predictions were necessarily guesses about the upcoming target color. In the prospective blocks, we therefore could not use only the "correct" targetpreceding judgments, because correct and incorrect predictions could not be discriminated on the basis of an objective performance criterion, as in the retrospective blocks.
Results

Color recollections and color predictions In a first analysis, we tested whether participants correctly recollected the preceding target color in the retrospective blocks. This was the case. Participants correctly recalled the color of the preceding target in $94.5 \%$ (range: $M=$ $68.8 \%$ to $98.5 \%, S D=7.5$ ) of the trials. By contrast, in the prospective blocks, the participants predicted that the upcoming target had the same color as in a preceding trial in only $51.4 \%$ of the trials (range: $M=41.2 \%$ to $70.2 \%, S D=7.3$ ). This means that in the prospective blocks, the likelihood of a judgment in accordance with a preceding target was not higher than chance (in our case, $50 \%), t<1$, and it was significantly lower than in the retrospective blocks, $t(15)=17.71, p<.01$. Together, these results indicate that our participants understood the two different blocked tasks and did not confuse them: They neither falsely recollected the target colors instead of making a prediction in the prospective blocks, nor did they falsely predict upcoming colors instead of recollecting the preceding targets in the retrospective blocks. Also, judging from the nonsignificant difference between the color discrimination times in the two tasks (retrospective blocks, $M=$ $948 \mathrm{~ms}$; predictive blocks, $M=909 \mathrm{~ms}), t(15)<1$, the tasks were about equally demanding.

As might be expected, in the prospective blocks, the participants' likelihood of a correct judgment about the color of the upcoming target was low, $49.3 \%$ (range: $M=46.5 \%$ to $53.9 \%)$, and therefore near chance level (50\%), from which it did not significantly differ, $t(15)=1.18, p=.23$.
Table $3 F$ values and $p$ levels of significance of a repeated measures ANOVA of the RTs and error rates of Experiment 2, for main effects of distractortarget position, judgment-target similarity, and type of targetpreceding judgment, as well as the corresponding interactions, for all data from the retrospective blocks, regardless of whether or not a recollection of the preceding target color was correct

\begin{tabular}{lll}
\hline Main Effect/Interaction & $F$ & $p$ \\
\hline Reaction Times & & \\
Distractor-target positions & 120.97 & $>.01$ \\
Target-judgment similarity & 31.10 & $>.01$ \\
Target-preceding judgment & 1.90 & .19 \\
Distractor-target position $\times$ Similarity & 15.72 & $>.01$ \\
Distractor-target position $\times$ Target-preceding judgment & 0.03 & .86 \\
Target-preceding judgment $\times$ Similarity & 4.37 & .05 \\
Distractor-target position $\times$ Target-preceding judgment $\times$ Similarity & 0.78 & .39 \\
Error Rates & & \\
Distractor-target positions & 4.58 & .05 \\
Target-judgment similarity & 0.51 & .49 \\
Target-preceding judgment & 11.28 & $>.01$ \\
Distractor-target position $\times$ Similarity & 1.11 & .31 \\
Distractor-target position $\times$ Target-preceding judgment & 1.77 & .20 \\
Target-preceding judgment $\times$ Similarity & .16 \\
Distractor-target position $\times$ Target-preceding judgment $\times$ Similarity & 2.22 & .56 \\
\hline
\end{tabular}


Target search Figure 3 shows capture effects for the correct responses observed in blocks with prospective and retrospective color judgments, separately for the two different distractor colors (similar or dissimilar to the judged target color; white and black histograms), and Table 4 shows the mean RTs and ERs for all conditions. Out of all trials, 4.2\% were eliminated from analyses because their RTs differed from the individual mean correct RTs by more than two standard deviations.

The main ANOVA of the correct median RTs was based on the variables target-preceding judgment (prospective vs. retrospective block), distractor-target positions (SP vs. DP), and judgment-target similarity (color similar vs. color dissimilar). (The main ANOVA was also repeated without the participant with the low recollection of only $68.8 \%$ correct judgments. After the exclusion of this participant, the remaining 15 participants' correct recollections ranged from $M=88.8 \%$ to $98.4 \%$. The $F$ values and significances of this ANOVA did not differ from the results of the analyses of all participants. The results after the exclusion are given in brackets below.)

In the RTs, there was again a strong capture effect: RTs were faster on SP trials $(M=703 \mathrm{~ms})$ than on DP trials $(M=755 \mathrm{~ms}), F(1,15)=128.30, p<.01[F(1,14)=$ $140.16, p<.01]$. Analogous to the stronger capture by word-similar colors in Experiment 1, this spatial capture effect of the distractors was significantly larger for distractors that were similar (and for targets that were dissimilar) to the judgment (capture effect $=64 \mathrm{~ms}$ ) than

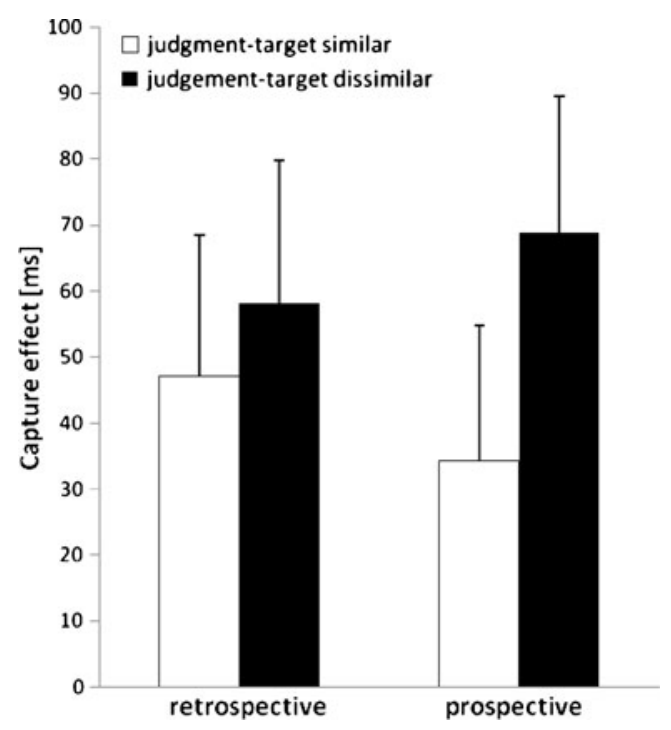

Fig. 3 Experiment 2's mean capture effects (calculated as RT in the DP conditions minus RT in the SP conditions) plus their corresponding standard errors (line bars), separately for the retrospective and prospective blocks and for the two different target colors (judgment-target similar [judgment-distractor dissimilar] or judgment-target dissimilar [judgment-distractor similar]; white and black histograms) for distractors that were dissimilar (and targets that were similar) to the judgment (capture effect $=41 \mathrm{~ms}$ ): judgment-target similarity $\times$ distractor-target position interaction, $F(1,15)=10.57, p<.01[F(1,14)=8.45, p<$ .01]. The capture effect was reliable in both the judgmenttarget similar and judgment-target dissimilar conditions [both $t \mathrm{~s}(15)>8.90$, both $p \mathrm{~s}<.01$ ].

In line with the automatic priming account, whether the judgments were predictions of the upcoming target color or whether they were recollections of the preceding target had an effect on neither attentional capture by the distractors in general (target-preceding judgment $\times$ distractor-target position interaction, $F<1$ $[F<1])$ nor the larger capture effect by judgment-similar as compared to judgment-dissimilar distractors (target-preceding judgment $\times$ distractor-target position $\times$ judgmenttarget similarity interaction: $F(1,15)=2.70, p=.12[F(1,14)$ $=2.56, p=.13]$ ).

Additional effects Analogous to the word-target similarity effect in Experiment 1, there was a main judgment-target similarity effect (or within-trial correspondence effect based on the similarity of the targets with WM content), with faster searches for judgment-similar targets $(M=709 \mathrm{~ms})$ than for judgment-dissimilar targets $(M=749 \mathrm{~ms})$ : main effect of judgment-target similarity, $F(1,15)=33.35, p<$ $.01[F(1,14)=28.37, p<.01]$. It indicated that within-trial correspondence with WM content overall facilitated responses, in addition to its modulating effect on attention capture. This overall within-trial correspondence effect for judged target colors (assessed as RT[judgment-target dissimilar] minus RT[judgment-target similar]) tended to be stronger in prospective blocks (similarity effect = $49 \mathrm{~ms}$ ) than in retrospective blocks (similarity effect = $30 \mathrm{~ms}$ ), possibly because of differences between the prediction and the recollection tasks, such as influences on decisions (see below): target-preceding judgment $\times$ judgment-target similarity interaction, $F(1,15)=3.59, p=$ $.08[F(1,14)=3.38, p=.09]$. Finally, the main effect of target-preceding judgment was not significant, $F(1,15)=$ $1.80, p=.20(F<1)$.

Error rates We found only a tendency toward attentional capture, with lower ERs in SP $(\mathrm{ER}=3.8 \%)$ than in DP (ER = $4.6 \%$ ) conditions: main effect of distractor-target position, $F(1$, $15)=3.39, p=.09[F(1,14)=3.14, p=.10]$. In addition, the retrospective task was evidently easier than the prospective task, which was reflected in a lower ER in the retrospective $(\mathrm{ER}=3.4 \%)$ than in the prospective $(\mathrm{ER}=5.1 \%)$ task: $F(1$, $15)=10.89, p<.01[F(1,14)=11.11, p<.01]$.

Additional analyses: Block order To test whether block order qualified our major conclusions, one further analysis 
Table 4 Mean RTs and ERs (plus standard deviations in parentheses) as a function of distractor-representation similarity (distractor color dissimilar vs. similar to the representation), target-preceding judgment (prediction vs. recollection), and target-distractor position (same position [SP] vs. different position [DP]) in Experiment 2

\begin{tabular}{lllll}
\hline Distractor Color & Target-Preceding Judgment & Position & RT (SD) & ER (SD) \\
\hline Dissimilar to the . . & $\ldots$ prediction & SP & $680(82)$ & $4.4(4.5)$ \\
& & DP & $714(81)$ & $6.4(5.3)$ \\
& $\ldots$ recollection & SP & $697(85)$ & $3.0(2.9)$ \\
Similar to the ... & . . prediction & SP & $744(86)$ & $3.3(3.5)$ \\
& & DP & $712(75)$ & $4.4(3.3)$ \\
& & SP & $781(91)$ & $5.1(5.7)$ \\
& DP & $721(86)$ & $3.4(4.4)$ \\
& & & $779(88)$ & $3.7(4.0)$ \\
\hline
\end{tabular}

included all of the above-mentioned variables plus the between-participants variable block order $(r-p-r-p, p-r-p-$ r). Block order interacted significantly with the type of target-preceding judgment (recollection vs. prediction), $F(1$, $15)=6.01, p<.05$. This interaction reflected a facilitation of RTs in the prospective blocks $(M=728 \mathrm{~ms})$ as compared to the retrospective blocks $(M=765 \mathrm{~ms})$ that was only present with block order $\mathrm{p}-\mathrm{r}-\mathrm{p}-\mathrm{r}$, not with block order $\mathrm{r}-\mathrm{p}-$ $\mathrm{r}-\mathrm{p}$ (prospective blocks, $M=718 \mathrm{~ms}$; retrospective blocks, $M=713 \mathrm{~ms})$. The main effect of block order was not significant, $F<1$, and it did not lead to any further significant interactions with the major variables of interest, all $F \mathrm{~s}<1$.

Intertrial priming of target predictions? In the prospective blocks, the independence between the participants' predictions and the preceding trials' target colors allowed us to test whether intertrial priming of the current prediction of target color by the preceding trial's target color affected attention shifts to the target in addition to the within-trial correspondence between target colors and judgments. An ANOVA of the correct median RTs of the prospective blocks, with the variables intertrial priming of the prediction by the preceding target color (predicted target color same as the preceding target color vs. predicted target color not same as the preceding target color), distractor-target positions (SP vs. DP), and within-trial judgment-target similarity (judgmenttarget color similar vs. dissimilar) showed that the capture effect was the same in trials on which the current distractor was intertrial-primed (and the prediction and the target were not primed) by the preceding target color (capture effect $=$ $53 \mathrm{~ms}$ ) as in trials on which the current distractor was not intertrial-primed (and the prediction and the target were primed; capture effect $=48 \mathrm{~ms}$ ): intertrial priming $\times$ distractor-target positions interaction, $F<1(F<1)$.

However, intertrial priming modulated the within-trial similarity or correspondence effect: intertrial targetprediction priming $\times$ within-trial judgment-target similarity interaction, $F(1,15)=8.70, p<.01[F(1,14)=9.15, p<$ .01]. The judgment-target similarity effect of faster responses for targets with a predicted color was almost twice as large when the target color from the previous trial had been repeated [judgment-target similarity effect $=$ $62 \mathrm{~ms} ; t(15)=4.61, p<.01]$ than when it had changed, and the current prediction and target were not primed by the preceding target's color [judgment-target similarity effect $=33 \mathrm{~ms} ; t(15)=3.26, p<.01]$. The main effect of intertrial priming, $F<1(F<1)$, and the three-way interaction, $F(1$, $15)=3.23, p=.09[F(1,14)=2.16, p=.16]$, were nonsignificant. For the results, see also Table 5.

\section{Discussion}

Our findings again suggested that automatic priming accounts for temporary changes in attentional control settings. We found that predicted colors as well as recollected colors, both of which were represented by our participants during a judgment at the beginning of a trial, facilitated capture by a distractor with a color similar to that in the preceding judgments. Yet, if anything, only the

Table 5 Mean RTs (plus standard deviations in parentheses) as a function of intertrial target color priming (yes [color of target repeats across trials] vs. no [color of target switches across trials]), prediction of the target color (correct $[=$ distractor dissimilar $]$ vs. incorrect $[=$ distractor similar]), and target-distractor position (same position [SP] vs. different position [DP]) in the prediction-judgment blocks of Experiment 2

\begin{tabular}{llll}
\hline Intertrial Priming & Prediction & Position & RT (SD) \\
\hline Yes & Correct (= d. dissimilar) & SP & $668(74)$ \\
& & DP & $713(95)$ \\
& Incorrect (= d. similar) & SP & $727(83)$ \\
& & DP & $778(82)$ \\
No & Correct (= d. dissimilar) & SP & $693(90)$ \\
& & DP & $721(79)$ \\
& Incorrect (= d. similar) & SP & $701(75)$ \\
& & DP & $779(97)$ \\
\hline
\end{tabular}

d. = distractor (the distractor had a color similar to the prediction when the target prediction was incorrect, and it had a color dissimilar to the prediction when the target prediction was correct). 
participants' predicted colors, not the recollected colors, affected the participants' expectancies of the most likely color of the upcoming target. Therefore, if the participants had strategic control over the selection of their temporary attentional control settings, we should have found a stronger capture effect from the distractors with a predicted target color in the prospective blocks, but not one from the distractors with a recollected target color in the retrospective blocks. In fact, the recollected colors were uncorrelated with the current colors, and therefore of little benefit for the selection of the attentional control settings for the upcoming target. The participants were also aware of this fact, as was evident from their very different judgments in the retrospective and prospective blocks.

A further interesting finding in Experiment 2 was that when the predicted color primed the attentional control settings, intertrial priming by a preceding target color had no additional modulating effect on spatial capture effects. This result resembles that from Experiment 1 (in which capture effects were actually even slightly lower if the distractor color was primed by the preceding target color) and is perfectly in line with the finding that cuing the target's dimension (whether the target is a shape or a color stimulus) in visual search can completely eliminate intertrial priming effects (cf. Fecteau, 2007). However, most prior studies have found that intertrial priming can boost attentional capture (cf. Becker, 2010; Becker, Ansorge, \& Horstmann, 2009), sometimes in addition to a word that cues the relevant target features (cf. Leonard \& Egeth, 2008; Müller, Reimann, \& Krummenacher, 2003). Regularly, such intertrial priming effects even accumulate across trials (cf. Maljkovic \& Martini, 2005; Maljkovic \& Nakayama, 1994; but see Leonard \& Egeth, 2008). At a first glance, our findings seem to contradict these results, as well as the interpretation that intertrial priming is automatic (e.g., Belopolsky et al., 2010; Kristjánsson \& Campana, 2010).

In our view, however, the present findings are not in conflict with the prior results. Much like a preceding target color, the representations of our participants automatically primed the dominant feature in the attentional-control settings. Thus, it is possible that in prior intertrial-priming studies, the participants represented the last-seen target color at the beginning of the majority of the trials, and that these representations were responsible for automatic priming of the attentional control settings (cf. Wolfe et al., 2003). Concerning the second issue - accumulation of color contingencies across many trials (cf. Maljkovic \& Martini, 2005) - we can only speculate. On a superficial view, if the participants' color predictions can overpower the representations of the last-seen target color, an impact of accumulated knowledge about the target features on attention capture seems impossible. However, one possibility is that evidence for the accumulation of intertrial priming of attention capture across more than two successive primed trials in previous studies actually reflected a prediction or an expectancy of the participants about the target color. In this view, Maljkovic and Martini might have measured an effect of the participants' predictions of the upcoming target color on attentional capture, but without a sufficiently sensitive procedure to demonstrate this (cf. Wolfe et al., 2003).

A final observation from Experiment 2 that deserves a brief discussion was the nonsignificant tendency for a numerically stronger capture effect (DP - SP performance) between the judgment-target-similar and the judgmenttarget-dissimilar conditions in the present experiment's prospective relative to its retrospective blocks. This result is interesting, because it suggests that a slight additional effect of strategic selection of the attentional control settings made it even more likely that the attentional control settings in the prospective blocks of Experiment 2 would correspond to the color predictions. Why an analogous additional strategic effect was not found in Experiment 1 (cf. Figs. 2 and 3) is not entirely clear, but several explanations are conceivable. In general, this small difference between the experiments suggests that the participants' own predictions were in fact not the same as those presented by the color word cues. Maybe it was easier to set up a nonverbal color template without a word cue in Experiment 2 than to represent word information in a target template (cf. Wolfe, Horowitz, Kenner, Hyle, \& Vasan, 2004). Maybe the participants' personal predictions about the target colors in Experiment 1 were also sometimes at variance with the external color cues in Experiment 1, but naturally not in Experiment 2, where no color cues were used. Whatever the exact reason, as a consequence, a slight additional effect of the strategic selection of attentional control settings might have been more likely in Experiment 2's prospective blocks than in Experiment 1's informative blocks. Note, however, that the corresponding three-way interactions between capture, block, and word-target or judgment-target similarity were far from significant in both experiments, and that the major significant, robust finding in both experiments was the reliable influence of automatic priming of attentional control settings by the participants' color representations.

\section{Experiment 3}

The primary aim of Experiments 1 and 2 was to demonstrate that it is possible that word cues or represented colors automatically rather than strategically affect temporary changes of attentional control settings. However, admittedly, the experiments do not provide compelling 
evidence that word cues will usually or necessarily effect such changes in attentional settings in a purely automatic fashion. First, and most importantly, neither a 75\%-correct color word cue (as in Exp. 1) nor the participants' own predictions concerning a factually unpredictable color (in Exp. 2) were optimal for convincing the participants of the benefits of strategic use of the color words. Hence, it is unclear whether automatic priming can indeed be demonstrated for capture effects of 100\%-valid word cues (e.g., Fecteau, 2007; Leonard \& Egeth, 2008)

Secondly, it is worth noting that the colors in Experiments 1 and 2 were nominally irrelevant for the task, because the response was defined by the orientation of a single line. Whereas some may regard the observed results as particularly strong evidence for automatic priming by color announcements (because rendering color irrelevant would have diminished all color-related effects and would have made it harder to find automatic priming by color words), others could argue that this may have compromised the strategic use of color words: Color words may only have been able to automatically bias attention because participants tried to universally ignore all colorrelated manipulations and to focus instead on searching for a target within a different stimulus dimension (shape). Although the results of Experiments 1 and 2 indicated that color announcements and judgments nevertheless modulated attention, it might still be regarded as an open question whether participants can ignore color representations when the target is defined on the same stimulus dimension (color) as the distractor and when the participants know the color of the upcoming target.

Experiment 3 was designed to address these questions. Deviating from the previous experiments, we used $100 \%$ informative color word cues in all conditions of Experiment 3. In one condition, the word cue validly indicated the upcoming target color. For example, if participants searched for red and green targets, the word "red" informed with $100 \%$ certainty that the next target was red, and the word "green" informed with $100 \%$ certainty that the next target was green. These were the target-congruent cues. By contrast, in the other, target-incongruent condition, the same targets were announced with $100 \%$ certainty by the opposite color words, so that "red" predicted with $100 \%$ validity that the target was green, while "green" indicated with $100 \%$ validity that the target was red. If participants used these word cues strategically, it should not matter which exact color word was used to inform the participants with $100 \%$ certainty about the next target color-for example, whether the target-congruent word "red" was used to inform the participants that the next target would be red, or whether the target-incongruent (and distractorcongruent) word "green" was used to inform that the next target would be red. As long as the word announced the next target's color with $100 \%$ certainty, and as long as there was enough time to translate the word into a search template for the next target color, the targets should be found with equal efficiency, and the distractors should be ignored to similar extents after target-congruent/distractor-incongruent and after target-incongruent/distractor-congruent cues.

In contrast to this prediction, if a word cue can (also) automatically prime the attentional capture by a relevant color of the top-down search set, it should be more difficult to search for the target after a distractor-congruent cue than after a target-congruent cue, because the distractorcongruent cue would automatically prime attention toward the distractor, whereas the target-congruent cue would automatically prime attention toward the target (cf. Theeuwes et al., 2006).

Deviating from the previous experiments, in Experiment 3 , color was rendered the target-defining feature and was clearly distinct from the response-defining feature: Each search display always contained two bars. One of the bars was always gray, whereas the other was one of two colors-for example, either red or green. Participants were instructed to search for the colored bar (red or green) and to report its orientation with a buttonpress. All displays also contained an irrelevant color distractor, which consisted of a colored circular outline that surrounded either the colored target bar (SP condition) or the gray nontarget bar (DP condition). A second circular outline was colored gray and presented at the alternative position (see Fig. 4). Compared with those in the previous experiments, the displays in Experiment 3 were thus more ambiguous with respect to the location of the

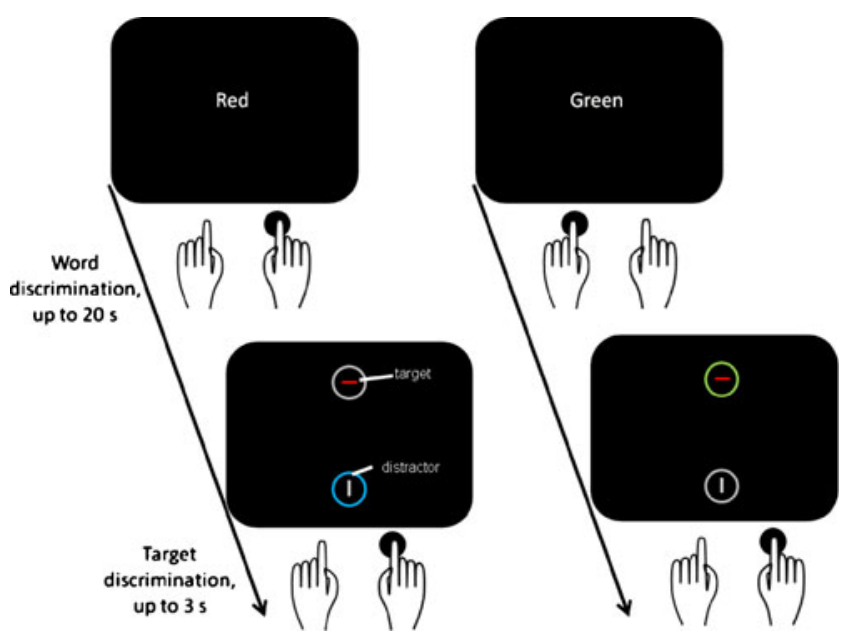

Fig. 4 Schematic examples of the sequences of events in a DP (different distractor-target position) trial (on the left) and of an SP (same distractor-target position) trial (on the right) of Experiment 3, with a target-congruent cue (on the left) and a target-incongruent cue (on the right). The arrow depicts the direction of time. Also depicted are buttonpresses to confirm the identity of the cue and start target search, and to discriminate the target. (Note that the pertaining mapping of color words to responses was also repeated at the beginning of the trial [not depicted].) Stimuli are not drawn to scale 
target, and participants were encouraged to adopt attentional control settings for a particular cued color on each trial (because the target was defined by this color). A possible exception was those trials on which the target and nontarget lines had identical orientations (i.e., both vertically or both horizontally oriented). On these trials, it might have been possible to determine the correct response without deploying spatial attention to the target color. This possibility was investigated in Experiment 3 by assessing the effects of same versus different target and distractor line orientations on attention.

If the automatic priming by color representations in Experiments 1 and 2 was due to the fact that participants did not explicitly search for a particular color, we should see no evidence for automatic priming by color words in Experiment 3; that is, we would expect that the distractor's capture effects would be no larger in target-incongruent/ distractor-congruent blocks than in target-congruent/distractorincongruent blocks (especially, perhaps, on those trials in which the line orientations of the target and distractor were identical). On the other hand, if even 100\%-informative color word cues can automatically trigger representations that bias attention toward color-similar items, then search should be slowed in target-incongruent blocks, and spatially specific capture effects (DP - SP) of the distractors should be larger in these blocks than in the targetcongruent blocks.

Larger distractor capture effects following from targetincongruent cues can, however, only be clearly attributed to automatic word priming if participants use the color cues to the same extent in both blocks. To rule out that greater task difficulty in the target-incongruent blocks would discourage participants from using the target-incongruent word cues, we included a control condition. On half of all trials in the target-congruent and target-incongruent blocks, we presented the distractor (a circular outline) in an irrelevant color that was unrelated to the present target and that was not part of the top-down search set. For example, if the participants searched for red and green targets, the distractor was blue on half of all trials. If participants were less inclined to process the target-incongruent word cue and to translate it into an appropriate search set than to read and use the easier target-congruent word cue, we should find larger distraction costs by these irrelevant colored distractors in the target-incongruent than in the targetcongruent blocks. On the other hand, if participants process the word cue in the target-incongruent conditions, too, then in the target-incongruent conditions, the cue-congruent distractor (e.g., red or green) should interfere more with search than the irrelevant and cueincongruent (e.g., blue) distractor, which in turn should show the same level of interference after targetcongruent and target-incongruent cues.
Method

Participants A group of 16 volunteers (13 female, 3 male; ages 20-38 years, mean age 23.6 years) received course credit to participate in this study. The participants had normal or corrected-to-normal vision.

Stimuli and procedure These were the same as before, with the following exceptions (see also Fig. 4). Firstly, different participants had to search for either blue (CIE color coordinates $=.157 / .107$ ) and green target bars, red and green target bars, or red and blue target bars (balanced across participants). The two target colors were realized equally often and were presented in a pseudorandom sequence of trials. Thus, the target bars were themselves colored rather than their backgrounds, as in the prior experiments. Secondly, each target bar was shown simultaneously with a gray vertical or horizontal shape-distractor bar. Across trials, target and distractor bar orientations were uncorrelated. (As before, if the target was above the screen center, the distractor was below it, and if the target was below the screen center, the distractor was above it.) Thirdly, target and distractor bars (both $0.08^{\circ}$ wide and $0.8^{\circ}$ long) were both surrounded by circles. The circles had a diameter of $1.0^{\circ}$ and stroke widths of $0.08^{\circ}$, and they were exactly centered on the same positions as the target and distractor lines, respectively. One of the circles was the color distractor, and the other circle was gray. The colored distractor circle had either a potentially relevant color (belonging to the set of target colors) or an irrelevant color (different from all searched-for target colors). On half of the trials, a relevant color distractor was presented, and it always had a color different from that of the current target: If the participants searched for red and green targets and the current target was red, for example, the relevant color distractor was green. On the other half of the trials, the color distractor had an irrelevant color. If the participants searched for red and green targets, for example, the irrelevant color distractor was blue. As before, the color distractor was presented equally often at the position of the target - this was the SP condition - or at the position of the distractor - this was the DP condition. Finally, a word cue correctly informed the participants about the target color on $100 \%$ of the trials. In the target-congruent blocks, the word cue always directly referred to the upcoming target color (e.g., the word "red" validly indicated that the target would be red). In target-incongruent blocks, the two color word cues always validly indicated the opposite target color (e.g., when the target could be red or green, the word "green" indicated that the target would be red, and the word "red" indicated that the target would be green). Distractors with a possible target color always had the color opposite the target color, so that the target-incongruent cues that were shown before a relevant 
color distractor were also distractor-congruent word cues. In trials with an irrelevant distractor, the target-incongruent cues were also distractor-incongruent (i.e., the cues referred to a color that was not present in the display).

Design The experiment consisted of two conditions that only differed with respect to the congruence between color cue and target color. In the target-congruent blocks, color cues denoted the predicted target color. In the targetincongruent blocks, color cues predicted the target color with a word that denoted an alternative color.

Again, the experiment consisted of four miniblocks: two blocks with target-congruent and two with targetincongruent cues. Congruent and incongruent blocks alternated (block sequence: congruent-incongruent-congruentincongruent, abbreviated "c-i-c-i"; or incongruent-congruentincongruent-congruent, abbreviated " $\mathrm{i}-\mathrm{c}-\mathrm{i}-\mathrm{c}$ "), with the block orders balanced across participants. Prior to the start of every block, participants were informed about which color word announced which target color in the upcoming block, and participants were encouraged to use the informative color word cue in all blocks to guide their target localization. A brief practice phase preceded the experiment. Each of the four miniblocks included 128 trials: four repetitions of the 32 combinations of 2 target colors $\times 2$ target orientations (vertical or horizontal) $\times 2$ target positions (above or below screen center) $\times 2$ cue colors (relevant or irrelevant) $\times 2$ distractor-target positions (SP or DP).

\section{Results}

Color word discrimination At the beginning of every trial, we informed the participants correctly about the color of the subsequent target on $100 \%$ of the trials. We tested whether the participants correctly discriminated this color cue. Accuracy for the color word discrimination was high in target-congruent (99.9\%) and in target-incongruent $(99.8 \%)$ blocks and did not significantly differ between blocks, $t<$ 1. This meant that the participants correctly discriminated and read the color cues in virtually all trials. As might be expected, the color word discrimination took slightly longer for the target-incongruent cues $(M=829 \mathrm{~ms})$ than for the target-congruent cues $(M=763 \mathrm{~ms}), t(15)=1.37, p=.09$.

Target search One participant had to be excluded because of too high an ER on at least some of the trials. See also Fig. 5 and Table 6 for the results. Out of all trials, 5.5\% were eliminated from the analyses because RTs differed from the individual mean correct RT by more than two standard deviations.

The results are based on a repeated measures ANOVA restricted to trials with correct color word discriminations, and based on the variables word cue announcement (target-

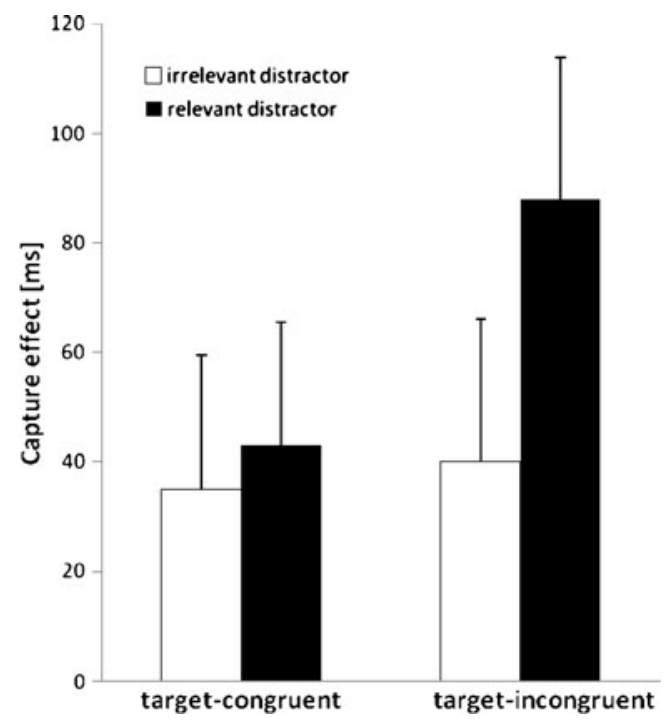

Fig. 5 Experiment 3's mean capture effects (calculated as RT in the $\mathrm{DP}$ conditions minus RT in the SP conditions) plus their corresponding standard errors (line bars), separately for the targetcongruent and target-incongruent cue announcements and for the two different distractor types (irrelevant, and hence always incongruent to the cues, or relevant, and hence incongruent to the cues in the targetcongruent conditions vs. congruent to the cues in the targetincongruent conditions; white and black histograms)

congruent vs. target-incongruent block), distractor-target positions (SP vs. DP), color distractor type (relevant vs. irrelevant), and distractor line type (same as or different from the target). Again, we found a significant capture effect of the distractors. RTs were faster on SP trials $(M=$ $604 \mathrm{~ms})$ than on DP trials $(M=655 \mathrm{~ms}), F(1,14)=$ 14.38, $p<.01$. As expected, spatial capture was significantly greater for the relevant color distractors (capture effect [RT in DP trials minus RT in SP trials] = $65 \mathrm{~ms}$ ) than for the irrelevant color distractors (capture effect $=37 \mathrm{~ms}$ ) [color-distractor type $\times$ distractor-target position interaction, $F(1,14)=4.83, p<.05$ ], and it was greater in the target-incongruent (capture effect $=64 \mathrm{~ms}$ ) than in the target-congruent (capture effect $=38 \mathrm{~ms}$ ) blocks [word announcement $\times$ distractor-target position interaction, $F(1,14)=4.99, p<.05]$. Most importantly, we also found evidence for automatic priming of attentional capture. This was reflected in a significant three-way interaction of color distractor type, distractor-target position, and word cue announcement, $F(1,14)=9.89, p<.01$. A follow-up ANOVA of only the relevant distractors showed that the capture effect of the relevant distractors significantly increased from target-congruent/distractor-incongruent blocks (capture effect $=43 \mathrm{~ms}$ ) to target-incongruent/ distractor-congruent blocks (capture effect $=88 \mathrm{~ms}$ ). This was reflected in a significant two-way interaction of the variables word cue announcement (or block) and distractor-target position in this follow-up ANOVA, $F$ 
Table 6 Mean RTs and ERs (plus standard deviations in parentheses) as a function of distractor orientation (same as vs. different from target), distractor color (relevant vs. irrelevant), announcement (targetcongruent/relevant-distractorincongruent vs. targetincongruent/relevant-distractorcongruent), and target-distractor position (same position [SP] vs. different position [DP]) in Experiment 3

\begin{tabular}{|c|c|c|c|c|c|}
\hline Distractor Orientation & Distractor Color & Announcement & Position & $\mathrm{RT}(S D)$ & $\mathrm{ER}(S D)$ \\
\hline \multirow[t]{8}{*}{ Same as target } & \multirow[t]{4}{*}{ Relevant } & \multirow[t]{2}{*}{ Target congruent } & SP & $602(79)$ & $3.2(4.0)$ \\
\hline & & & DP & $623(87)$ & $3.0(3.8)$ \\
\hline & & \multirow[t]{2}{*}{ Target incongruent } & SP & $580(76)$ & $4.9(5.3)$ \\
\hline & & & DP & $657(122)$ & $4.1(5.3)$ \\
\hline & \multirow[t]{4}{*}{ Irrelevant } & \multirow[t]{2}{*}{ Target congruent } & SP & $593(74)$ & $3.0(3.7)$ \\
\hline & & & DP & 618 (109) & $4.9(5.0)$ \\
\hline & & \multirow[t]{2}{*}{ Target incongruent } & SP & $589(82)$ & $3.3(4.1)$ \\
\hline & & & DP & $626(130)$ & $4.3(4.0)$ \\
\hline \multirow[t]{8}{*}{ Different from target } & \multirow[t]{4}{*}{ Relevant } & \multirow[t]{2}{*}{ Target congruent } & SP & $621(84)$ & $2.6(3.7)$ \\
\hline & & & DP & 683 (109) & $6.1(6.5)$ \\
\hline & & \multirow[t]{2}{*}{ Target incongruent } & SP & $623(82)$ & $6.0(6.8)$ \\
\hline & & & DP & 724 (132) & $6.7(8.6)$ \\
\hline & \multirow[t]{4}{*}{ Irrelevant } & \multirow[t]{2}{*}{ Target congruent } & SP & $611(77)$ & $4.5(5.0)$ \\
\hline & & & DP & 657 (127) & $2.1(3.5)$ \\
\hline & & \multirow[t]{2}{*}{ Target incongruent } & SP & $614(76)$ & $4.9(5.5)$ \\
\hline & & & DP & 656 (124) & $6.0(6.1)$ \\
\hline
\end{tabular}

$(1,14)=9.89, p<.01$. In contrast, in a follow-up ANOVA of only the irrelevant distractors, no such increase was observed. The capture effect was barely affected by whether a target-congruent word (capture effect $=35 \mathrm{~ms}$ ) or a target-incongruent word (capture effect $=40 \mathrm{~ms}$ ) was used as a cue: $F<1$ for the word cue announcement $\times$ distractor-target position relations interaction.

In addition, we found a significant main effect of color distractor type, $F(1,14)=6.70, p<.05$, with faster responses when an irrelevant color distractor was present $(M=621 \mathrm{~ms})$ than in conditions with a relevant color distractor $(M=$ $639 \mathrm{~ms}$ ), and a significant main effect of distractor line type, $F(1,14)=75.66, p<.01$, with faster responses if the distractor line was of the same orientation as the target $(M=$ $611 \mathrm{~ms}$ ) than if it was of a different orientation from the target $(M=649 \mathrm{~ms})$. The variable distractor line type also interacted significantly with the variable color distractor type, $F(1,14)=5.12, p<.05$, and with the variable distractor-target position, $F(1,14)=5.36, p<.05$. These two interactions reflected a stronger distractor line type effect [RT (different orientations) - RT(similar orientations)] of the relevant color distractors (distractor line type effect $=47 \mathrm{~ms}$ ) than of the irrelevant color distractors (distractor line type effect $=29 \mathrm{~ms}$ ), and a stronger capture effect (DP - SP) for distractor lines of a different orientation from the target's (capture effect $=49 \mathrm{~ms}$ ) than for distractor lines with the same orientation as the target (capture effect $=26 \mathrm{~ms}$ ), respectively. Importantly, line orientation did not modulate differences in the capture effects between target-incongruent and targetcongruent blocks, indicating that target line orientation did not affect word priming of the distractors' capture effects, all other $F_{\mathrm{s}}<2.10$, all $p \mathrm{~s}>.17$.
A similar ANOVA of ERs led to no significant main effects or interactions, all $F_{\mathrm{S}}<3.10$, all $p \mathrm{~s}>.05$.

Additional analyses Again, we also tested in an ANOVA, with the same variables as above and the additional between-participants variable block order (c-i-c-i or $\mathrm{i}-\mathrm{c}-$ $\mathrm{i}-\mathrm{c}$ ), whether our major conclusions were affected by the block sequence. This was not the case. There were only significant interactions between block order and word cue announcement, $F(1,13)=14.45, p<.01$, as well as block order and distractor line type, $F(1,13)=5.45, p<.05$. The former interaction was due to an advantage in cue-targetcongruent as compared to cue-target-incongruent blocks [congruence effect: RT(target incongruent) - RT(target congruent)] only for the participants who started with the target-congruent announcements (congruence effect = $46 \mathrm{~ms}$ ). By contrast, for the participants who started with the target-incongruent announcements, we found an almost similarly strong facilitation in the incongruent relative to the congruent blocks (congruence effect $=-37 \mathrm{~ms}$ ). This means that whatever level of congruence was experienced and exercised first was easier for the participants. The second interaction reflected stronger interference by differently as compared to similarly oriented distractors and targets [interference effect $=\mathrm{RT}$ (different orientations $)-\mathrm{RT}$ (similar orientations) $]$ in $\mathrm{c}-\mathrm{i}-\mathrm{c}-\mathrm{i}$ (interference effect $=$ $47 \mathrm{~ms}$ ) versus $\mathrm{i}-\mathrm{c}-\mathrm{i}-\mathrm{c}$ sequences (interference effect $=$ $39 \mathrm{~ms})$. We do not know the reason for this interaction. Maybe it reflected differences between the participants. Importantly, however, the main effect of block order, $F<1$, and the remaining interactions with this variable, all $F \mathrm{~s}<$ 2.90 , all $p \mathrm{~s}>.11$, were not significant. 
In addition, it has been demonstrated that prior task experience heavily influences attentional control settings (Leber \& Egeth, 2006; Thompson, Underwood, \& Crundall, 2007), and therefore we tested the correct RT performance in only the first blocks as a function of the between-participants variable word cue announcement (and the remaining three within-participants variables, as in the initial ANOVA above). This ANOVA confirmed [by its significant three-way interaction between word cue announcement, distractor-target position, and color-distractor type, $F$ $(1,13)=6.12, p<.05]$ that only for the participants who started with the cue-target-incongruent conditions, the relevant and cue-similar distractor captured more attention (capture effect: $121 \mathrm{~ms}$ ) than the irrelevant distractor (capture effect: $50 \mathrm{~ms}$ ): distractor-target position $\times$ color-distractor type, $F(1,7)=8.58, p<.05$ (in a follow-up ANOVA of only those participants who started with a word-targetincongruent block). In contrast, for the participants who started with the word-target-congruent conditions, the relevant and cue-similar distractor captured about as much attention (capture effect: $32 \mathrm{~ms}$ ) as the irrelevant distractor (capture effect, $40 \mathrm{~ms}$ ): distractor-target position $\times$ color distractor type, $F<1$ (in a follow-up ANOVA of only those participants who started with a word-target-congruent block).

As in Experiments 1 and 2, we also tested whether intertrial priming of attentional capture by repeated target colors was or was not eliminated by the use of targetcongruent and/or target-incongruent word announcements. To that end, we ran a complementary ANOVA of the correct RTs, with the variables distractor-target position, color distractor type, word cue announcement, and intertrial priming (target color repetition $[n-1=n]$ vs. target color switch $[n-1 \neq n])$, with the data collapsed across different and similar target and distractor line orientations. Besides the aforementioned effects, there was a significant main effect of intertrial priming, $F(1,14)=9.13, p<.05$, with faster RTs for repeated target colors $(M=620 \mathrm{~ms})$ than for switched target colors $(M=637 \mathrm{~ms})$. However, no significant interactions between priming and one of the variables of interest - such as cue-target position, word cue announcement, or color distractor type-or between priming and one of the interactions of interest were found (for all interactions involving priming, $F \mathrm{~s}<2.90, p \mathrm{~s}>$ .11). See also Table 7 for the results of this analysis.

\section{Discussion}

In line with automatic priming of attentional control settings by the representation of a color, we found once more that color words at the beginning of a trial biased participants' search toward the announced color. If such a color word denoted a color similar to that of the distractor, attention was biased toward the distractor, as evidenced by stronger spatial capture effects of the distractor in this condition than when the word denoted a color dissimilar to that of the distractor. This was found in a comparison of capture effects between target-congruent/distractorincongruent word cue conditions and target-incongruent/ distractor-congruent word cue conditions. Taken together, the results demonstrate automatic color word priming of capture under conditions in which color was relevant for the task and the word cue indicated the target color with $100 \%$ certainty.

We found that capture effects were attenuated when the distractor line was of the same orientation as the target line.
Table 7 Mean RTs (plus standard deviations in parentheses) as a function of intertrial target color priming (yes [color of target repeats across trials] vs. no [color of target switches across trials]), distractor color (relevant vs. irrelevant), announcement (targetcongruent/relevant-distractorincongruent vs. targetincongruent/relevant-distractorcongruent), and target-distractor position (same position [SP] vs. different position [DP]) of Experiment 3

\begin{tabular}{lllll}
\hline Intertrial Priming & Distractor Color & Announcement & Position & RT (SD) \\
\hline Yes & Relevant & Target congruent & SP & $595(73)$ \\
& & & DP & $649(92)$ \\
& & Target incongruent & SP & $585(67)$ \\
& & & DP & $694(128)$ \\
& Irrelevant & Target congruent & SP & $587(67)$ \\
& & DP & $625(95)$ \\
& & Target incongruent & SP & $590(75)$ \\
& & DP & $636(127)$ \\
& Relevant & Target congruent & SP & $626(86)$ \\
& & DP & $656(103)$ \\
& & Target incongruent & SP & $607(86)$ \\
& & DP & $685(123)$ \\
& Irrelevant & Target congruent & SP & $614(80)$ \\
& & DP & $650(159)$ \\
& & Target incongruent & SP & $608(79)$ \\
& & DP & $646(128)$ \\
& & &
\end{tabular}


This might be taken as evidence that, on a proportion of the same-orientation trials, the response could be specified without attending to the target color. Importantly, however, whether the target was or was not of the same orientation as the target did not affect the overall pattern of a selectively increased capture effect by those relevant color distractors that were automatically primed by a preceding color word. Thus, same-orientation trials did not selectively affect color representations or the contents of the attentional control settings, but very likely facilitated responses in an attentionindependent manner. For example, the distractor lines might have facilitated the choice of the correct response by motor priming that took place in parallel to the attentional selection of targets by their colors (cf. Eriksen \& Eriksen, 1974). If attentional selection and motor activation occurred in parallel, a distractor line of the same orientation as the target would have facilitated the process of selecting the correct response, and thus also curtailed the attentionalcapture effect. On the other hand, it is also possible that distractor line orientation did not directly bypass attention, but that it was modulated by attention. Line orientation compatibility effects are expected to be stronger when attention is deployed to the distractor, because allocating spatial attention to the distractor will lead to processing of the orientation of the gray nontarget line inside the distractor, whereas the line orientation inside the distractor will mostly be ignored when attention is not deployed to the distractor (e.g., Becker, 2007; Starreveld, Theeuwes, \& Mortier, 2004; Theeuwes \& Burger, 1998). The finding of stronger distractor line effects for relevant color distractors and for distractors with stronger spatial interference effects is thus not unexpected.

Taken together, the results of the present experiment supported the assumption that color representations can automatically prime capture by a color distractor (cf. Theeuwes et al., 2006), and they show that this automatic priming effect can not only be observed under conditions with low cue (or color representation) validity, as were used in Experiments 1 and 2.

Two additional results of Experiment 3 also deserve a brief discussion. In the present experiment, we used relevant color distractors as well as irrelevant color distractors. The relevant color distractors had a color matching the set of searched-for target colors, and the irrelevant color distractors had a color that did not match the set of searched-for target colors. Many previous studies have shown that under these conditions, relevant or matching distractors capture attention more reliably or for longer than irrelevant or nonmatching distractors (e.g., Folk \& Remington, 1998). In contrast to this, no such top-downcontingent effect was found in the present experiment. In the present experiment, a stronger capture effect of the relevant or matching distractors than of the irrelevant or nonmatching distractors was restricted to the conditions in which a distractor-congruent word primed attention capture by the relevant distractors; however, when a targetcongruent word informed the participants about the color of the searched-for target, attention capture was the same for relevant and irrelevant distractors.

One possibility for this lack of more capture by matching distractors in the word-target-congruent conditions was that under conditions with two relevant colors, as were used here, attentional control settings are difficult to maintain, and even irrelevant distractors capture attention (Folk \& Anderson, 2010). On closer inspection, a second possibility becomes clear, too: The absence of more capture by the relevant than by the irrelevant distractors in the wordtarget-congruent blocks is probably just the flip-side of the stronger capture effect of the relevant distractor if this distractor was primed in the target-incongruent conditions: To the extent that capture by the relevant distractor could be primed by a distractor-congruent word, this capture should also be overcome if capture by the relevant target was primed by way of a target-congruent word. In this automatic-priming view, attentional capture was the same for relevant and for irrelevant distractors in the word-targetcongruent blocks because this capture effect reflected the residual capture effect of the colored distractors, which was due either to exogenous capture or to a top-down set for all colored stimuli under the present conditions.

Another interesting observation concerned the influence of intertrial priming of the target colors. Again, we found that a repetition of the target colors across subsequent trials facilitated finding the target. This was evident in a comparison with those trials on which the current target color switched from the preceding to the current trial. However, as is clear from the lack of an effect of intertrial priming on the distractor's capture effect, intertrial priming did not change attentional biases (e.g., from selecting red to selecting green, if the previous target had been green; cf. Maljkovic \& Nakayama, 1994). If that had been the case, we would have expected a significant interaction between intertrial priming of the target color and the variable distractor-target position (SP vs. DP), such that capture effects were attenuated in intertrial-primed target color conditions. Yet this interaction was not significant, and if anything, the capture effect of the distractors tended to be larger on trials with an intertrial-primed target color (capture effect $=62 \mathrm{~ms}$ ) than on trials in which the color of the target switched from the previous to the current trial (capture effect $=46 \mathrm{~ms}$ ). One possibility is that the word cues in the present experiments eliminated such intertrial effects on attentional capture by the distractors. The words served as primes, much as the colors of the targets on preceding trials had in previous studies. However, the word cues' priming of attentional capture probably completely 
overrode (or overshadowed) the intertrial priming of capture by repeated target colors, because the words were temporally closer to the targets and were presented for a longer duration than the targets.

\section{General discussion}

In the present study, we tested whether word cues at the beginning of a trial are used strategically or automatically. In Experiment 1, we found that a word cue biased attention capture by a color distractor similar to the word, regardless of how well the word informed about the probable target color. However, one could argue that the results with the word cues were misleading because the subjective expectancies of the participants might differ from the objective probability manipulations. If that was the case, the words would simply not have corresponded to our participants' expectancies.

To test whether expected or predicted target colors were strategically used for the setup of attentional control settings, we ran Experiment 2. In this experiment, at the beginning of every trial, we asked our participants which target color they predicted. Tentatively, in line with strategic use of such predictions, distractors that had a color similar to that in the predictions indeed captured more attention than did distractors with a color dissimilar from that in the predictions. However, on closer inspection, this boosted capture effect of distractors with a predicted color was also very likely due to automatic priming of capture. When we compared the capture effects of the predicted colors with the capture effects of recollected colors, we again found that any representation-similar distractor captured more attention than did a representationdissimilar distractor: For example, if participants recollected that the preceding target had been green, more capture was found by a green than by a red distractor. This was the case although (a) the preceding and the present trial's target colors were uncorrelated, and hence strategic use of the recollected colors for the control of the search set for the current target color was unlikely, and (b) the predictions of the participants about the current target color were accordingly uncorrelated with the colors of the preceding targets. Together, these results confirmed the conclusion from Experiment 1 that color representations automatically primed attention capture by a representation-similar distractor.

Both Experiments 1 and 2, however, could also be jointly criticized because participants were not able to predict the target colors with certainty in any of the conditions. Note that even the informative color words in the first experiment indicated the true color of the upcoming target with only $75 \%$ validity. Even worse, participants had chance-level accuracy when predicting the target colors in Experiment 2. Therefore, we ran Experiment 3, in which color words indicated the color of the upcoming target with $100 \%$ certainty. Yet, even in this experiment, we found that the word cues primed attention capture by the distractors in an automatic and nonstrategic fashion. If a target-congruent word was used to inform about the target color, attention capture by the color distractors was weaker than if a distractor-congruent word was used to inform about the target color. For example, if the word cue "red" informed that the target color was red, attention capture by a green distractor was weaker than if the word cue "green" informed that the target color was red. (Because the capture by an irrelevant color distractor, such as a blue distractor when the color word cues were "red" and "green," was not affected by the manipulation of cuetarget congruence, the effect was due to automatic priming of capture instead of to a failure to use the targetincongruent/distractor-congruent word cues at all.)

Together, the facilitating effects of any color representation on subsequent capture by a word-similar or representationsimilar color stimulus confirmed the suspicion that automatic priming could determine the temporary feature template that is used in an attentional control setting (cf. Moore \& Weissman, 2010, 2011). Related findings in support of automatic priming of attentional control settings have been reported in the intertrial-priming literature (for a recent review, see Kristjánsson \& Campana, 2010) and in studies of WM influences on attention capture (cf. Belke, Humphreys, Watson, Meiyer, \& Telling, 2008; Moores, Laiti, \& Chelazzi, 2003).

By the same token, our results advise caution when using word cues (e.g., the word "green") or other instructional cues with a particular meaning (such as the picture of a green stimulus) at the beginning of a trial to inform about the target features (cf. Fecteau, 2007; Leonard \& Egeth, 2008). These words or cues might automatically prime meaning-congruent attentional control settings (cf. Martens et al., 2011; Theeuwes et al., 2006). Therefore, caution is necessary if the modulation of attentional capture by an instructional cue or word is ascribed to the participants' strategic selection of the temporary attentional control settings. For example, if a word with a particular meaning, such as "green," is used to inform about the target on $100 \%$ of the trials, the word meaning could automatically prime a meaning-congruent attentional control setting for the denoted feature (see the present Exp. 3). Alternatively, the word could encourage the participants to strategically use the word for the specification of the attentional control settings for the upcoming target because of the word's utility as a $100 \%$-correct prediction of the target. Only if the same word were used with the same attentional control settings, but once with more and once 
with less predictive value for the identity of the searchedfor relevant target features, could it be decided whether the word automatically primed the attentional control settings or whether it was used strategically. An automatic-priming effect on attentional control settings should be the same for target-feature-informative and -uninformative primes, whereas strategic selection should be stronger with targetinformative than with -uninformative primes.

\section{Intertrial priming}

If asked to predict the upcoming target color, we also found that the participants predicted another color than the repeated target color from the last trial on half of the trials (Exp. 2). This means that the preceding target color evidently did not prime the participants' prediction of the upcoming target color. What is more, when we compared the within-trial priming of the attentional control settings by the participants' own target color predictions in Experiment 2 and by word discriminations in Experiments 1 and 3 with the respective intertrial priming of the attentional control settings by the preceding trial's target colors in the very same conditions, we found that the color-specific attentional control settings were virtually solely primed by the color predictions or by the color word cues within a trial, and that the intertrial priming of preceding target colors on current target colors had little additional influence on the attentional control settings.

These findings do not necessarily put in question the standard explanation of intertrial priming effects of colorspecific attentional capture as a purely stimulus-driven process. They only show that the representation of the recollected target color is as powerful in priming attentional control settings as is the predicted upcoming target color (for related results with encoded dimensions, see Müller, Krummenacher, \& Heller, 2004). According to this line of argument, the words were simply temporally more recent or more relevant than the preceding target colors, and therefore had a greater priming effect than the preceding trial's target colors.

\section{Perspective}

The present study suggests a division of labor between strategic top-down control of attentional control settings as a factor determining the relevance of features for the intermediate- to long-term duration of an experiment, on the one hand, and automatic priming of a particular feature template within these controls settings as a factor responsible for the temporary adaptation of the attentional control settings, on the other hand. The underlying reason for this division of labor could be that the strategic revision of the attentional control settings takes more time than does automatic priming. As a consequence of this, quick adjustments of attentional control settings via automatic priming could be beneficial for the reoccurring short-term changes of the specific dominating feature in an attentional control setting, although these quick adjustments carry the cost of an occasionally less-than-optimal precision in top-down attentional control. It remains to be seen whether this division of labor can be validated in future research.

Author note Thanks to four anonymous reviewers and to Hermann Müller for excellent comments on a previous version of the present article, and to Katharina Sehling for help with the data collection. This research was supported by Deutsche Forschungsgemeinschaft Grants An 353/2-1, to U.A., and 353/5-1, to U.A., Ingrid Scharlau, and Werner Klotz, and by travel grants from the University of Queensland, Brisbane, Australia, and the University of Vienna, Austria.

\section{References}

Ansorge, U., Kiss, M., Worschech, F., \& Eimer, M. (2011). The initial stage of visual selection is controlled by top-down task set: New ERP evidence. Attention, Perception, \& Psychophysics, 73, 113122.

Ansorge, U., \& Heumann, M. (2003). Top-down contingencies in peripheral cuing: The roles of color and location. Journal of Experimental Psychology: Human Perception and Performance, 29, 937-948. doi:10.1037/0096-1523.29.5.937

Ansorge, U., \& Heumann, M. (2004). Peripheral cuing by abruptonset cues: The influence of color in S-R corresponding conditions. Acta Psychologica, 116, 115-143. doi:10.1016/j. actpsy.2004.01.001

Ansorge, U., \& Horstmann, G. (2007). Preemptive control of attentional capture by color: Evidence from trial-by-trial analysis and ordering of onsets of capture effects in RT distributions. Quarterly Journal of Experimental Psychology, 60, 952-975.

Bargh, J. A. (1992). The ecology of automaticity: Toward establishing the conditions needed to produce automatic processing effects. American Journal of Psychology, 105, 181-199.

Becker, S. I. (2007). Irrelevant singletons in pop-out search: Attentional capture or filtering costs? Journal of Experimental Psychology: Human Perception and Performance, 33, 764-787. doi:10.1037/0096-1523.33.4.764

Becker, S. I. (2008a). Can intertrial effects of features and dimensions be explained by a single theory? Journal of Experimental Psychology: Human Perception and Performance, 34, $1417-$ 1440. doi: $10.1037 / \mathrm{a} 0011386$

Becker, S. I. (2008b). The stage of priming: Are intertrial repetition effects attentional or decisional? Vision Research, 48, 664-684. doi:10.1016/j.visres.2007.10.025

Becker, S. I. (2010). The role of target-distractor relationships in guiding attention and the eyes in visual search. Journal of Experimental Psychology: General, 139, 247-265. doi:10.1037/ a0018808

Becker, S. I., Ansorge, U., \& Horstmann, G. (2009). Can intertrial priming account for the similarity effect in visual search? Vision Research, 49, 1738-1756. doi:10.1016/j.visres.2009.04.001

Becker, S. I., Folk, C. L., \& Remington, R. W. (2010). The role of relational information in contingent capture. Journal of Experimental Psychology: Human Perception and Performance, $36,1460-1476$. 
Belke, E., Humphreys, G. W., Watson, D. G., Meyer, A. S., \& Telling, A. L. (2008). Top-down effects of semantic knowledge in visual search are modulated by cognitive but not perceptual load. Perception \& Psychophysics, 70, 1444-1458. doi:10.3758/ PP.70.8.1444

Belopolsky, A. V., Schreij, D., \& Theeuwes, J. (2010). What is topdown about contingent capture? Attention, Perception, \& Psychophysics, 72, 326-341.

Bichot, N. P., Rossi, A. F., \& Desimone, R. (2005). Parallel and serial neural mechanisms for visual search in macaque area V4. Science, 308, 529-534. doi:10.1126/science.1109676

Burnham, B. R. (2007). Displaywide visual features associated with a search display's appearance can mediate attentional capture. Psychonomic Bulletin \& Review, 14, 392-422.

Duncan, J., \& Humphreys, G. W. (1989). Visual search and stimulus similarity. Psychological Review, 96, 433-458. doi:10.1037/ 0033-295X.96.3.433

Eriksen, B. A., \& Eriksen, C. W. (1974). Effects of noise letters upon the identification of a target letter in a non-search task. Perception \& Psychophysics, 16, 143-149. doi:10.3758/ BF03203267

Fecteau, J. H. (2007). Priming of pop-out depends upon the current goals of observers. Journal of Vision, 7(6), 1:1-11. doi:10.1167/ 7.6.1

Folk, C. L., \& Anderson, B. A. (2010). Target-uncertainty effects in attentional capture: Color-singleton set or multiple attentional control settings? Psychonomic Bulletin \& Review, 17, 421-426. doi:10.3758/PBR.17.3.421

Folk, C. L., \& Remington, R. W. (1998). Selectivity in distraction by irrelevant featural singletons: Evidence for two forms of attentional capture. Journal of Experimental Psychology: Human Perception and Performance, 24, 847-858.

Folk, C. L., \& Remington, R. W. (2008). Bottom-up priming of topdown attentional control settings. Visual Cognition, 16, 215-231.

Folk, C. L., Remington, R. W., \& Johnston, J. C. (1992). Involuntary covert orienting is contingent on attentional control settings. Journal of Experimental Psychology: Human Perception and Performance, 18, 1030-1044. doi:10.1037/0096-1523.18.4.1030

Gaissmaier, W., \& Schooler, L. J. (2008). The smart potential behind probability matching. Cognition, 109, 416-422.

Geyer, T., \& Müller, H. J. (2009). Distinct, but top-down modulable color and positional priming mechanisms in visual pop-out search. Psychological Research, 73, 167-176.

Hillstrom, A. P. (2000). Repetition effects in visual search. Perception \& Psychophysics, 62, 800-817. doi:10.3758/BF03206924

Kiefer, M. (2002). The N400 is modulated by unconsciously perceived masked words: Further evidence for a spreading activation account of N400 priming effects. Cognitive Brain Research, 13, 27-39.

Kristjánsson, Á., \& Campana, G. (2010). Where perception meets memory: A review of repetition priming in visual search tasks. Attention, Perception, \& Psychophysics, 72, 5-18. doi:10.3758/ APP.72.1.5

Kristjánsson, Á., \& Nakayama, K. (2003). A primitive memory system for the deployment of transient attention. Perception \& Psychophysics, 65, 711-724. doi:10.3758/BF03194808

Kristjánsson, Á., Sigurjónsdóttir, Ó., \& Driver, J. (2010). Fortune and reversals of fortune in visual search: Reward contingencies for pop-out targets affect search efficiency and target repetition effects. Attention, Perception, \& Psychophysics, 72, $1229-1236$.

Leber, A. B., \& Egeth, H. E. (2006). Attention on autopilot: Past experience and attentional set. Visual Cognition, 14, 565-583.

Leonard, C. J., \& Egeth, H. E. (2008). Attentional guidance in singleton search: An examination of top-down, bottom-up, and intertrial factors. Visual Cognition, 16, 1078-1091.
Maljkovic, V., \& Martini, P. (2005). Implicit short-term memory and event frequency effects in visual search. Vision Research, 45, 2831-2846.

Maljkovic, V., \& Nakayama, K. (1994). Priming of pop-out: I. Role of features. Memory \& Cognition, 22, 657-672. doi:10.3758/ BF03209251

Martens, U., Ansorge, U., \& Kiefer, M. (2011). Controlling the unconscious: Attentional task sets modulate subliminal semantic and visuo-motor processes differentially. Psychological Science, 22, 282-291.

Moore, K. S., \& Weissman, D. H. (2010). Involuntary transfer of a topdown attentional set into the focus of attention: Evidence from a contingent attentional capture paradigm. Attention, Perception, \& Psychophysics, 72, 1495-1509. doi:10.3758/APP.72.6.1495

Moore, K. S., \& Weissman, D. H. (2011). Set-specific capture can be reduced by pre-emptively occupying a limited-capacity focus of attention. Visual Cognition, 19, 417-444. doi:10.1080/13506285.2011.558862

Moores, E., Laiti, L., \& Chelazzi, L. (2003). Associative knowledge controls deployment of visual selective attention. Nature Neuroscience, 6, 182-189.

Müller, H. J., Krummenacher, J., \& Heller, D. (2004). Dimensionspecific inter-trial facilitation in visual search for pop-out targets: Evidence for a top-down modulable visual short-term memory effect. Visual Cognition, 11, 577-602.

Müller, H. J., Reimann, B., \& Krummenacher, J. (2003). Visual search for singleton feature targets across dimensions: Stimulus- and expectancy-driven effects in dimensional weighting. Journal of Experimental Psychology: Human Perception and Performance, 29, 1021-1035. doi:10.1037/0096-1523.29.5.1021

Nakayama, K., Maljkovic, V., \& Kristjánsson, Á. (2004). Short-term memory for the rapid deployment of visual attention. In M. S. Gazzaniga (Ed.), The cognitive neurosciences (3rd ed., pp. 387408). Cambridge, MA: MIT Press.

Olivers, C. N. L. (2009). What drives memory-driven attentional capture? The effects of memory type, display type, and search type. Journal of Experimental Psychology: Human Perception and Performance, 35, 1275-1291.

Olivers, C. N. L., \& Humphreys, G. W. (2003). Attentional guidance by salient feature singletons depends on intertrial contingencies. Journal of Experimental Psychology: Human Perception and Performance, 29, 650-657. doi:10.1037/0096-1523.29.3.650

Olivers, C. N. L., Meijer, F., \& Theeuwes, J. (2006). Feature-based memory-driven attentional capture: Visual working memory content affects visual attention. Journal of Experimental Psychology: Human Perception and Performance, 32, 12431265. doi:10.1037/0096-1523.32.5.1243

Savage, L. J. (1954). The foundations of statistics. New York: Wiley.

Soto, D., Heinke, D., Humphreys, G. W., \& Blanco, M. J. (2005). Early, involuntary top-down guidance of attention from working memory. Journal of Experimental Psychology: Human Perception and Performance, 31, 248-261. doi:10.1037/00961523.31.2.248

Starreveld, P. A., Theeuwes, J., \& Mortier, K. (2004). Response selection in visual search: The influence of response compatibility of nontargets. Journal of Experimental Psychology: Human Perception and Performance, 30, 56-78.

Theeuwes, J. (2010). Top-down and bottom-up control of visual selection. Acta Psychologica, 135, 77-99. doi:10.1016/j. actpsy.2010.02.006

Theeuwes, J., \& Burger, R. (1998). Attentional control during visual search: The effect of irrelevant singletons. Journal of Experimental Psychology: Human Perception and Performance, 24, 1342-1353. doi:10.1037/0096-1523.24.5.1342

Theeuwes, J., Reimann, B., \& Mortier, K. (2006). Visual search for featural singletons: No top-down modulation, only bottom-up priming. Visual Cognition, 14, 466-489. doi:10.1080/13506280500195110 
Thompson, C., Underwood, G., \& Crundall, D. (2007). Previous attentional set can induce an attentional blink with task-irrelevant initial targets. Quarterly Journal of Experimental Psychology, 60, 1603-1609. doi:10.1080/17470210701536468

Wolfe, J. M., Butcher, S. J., Lee, C., \& Hyle, M. (2003). Changing your mind: On the contributions of top-down and bottom-up guidance in visual search for feature singletons. Journal of Experimental Psychology: Human Perception and Performance, 29, 483-502. doi:10.1037/0096-1523.29.2.483
Wolfe, J. M., Horowitz, T. S., Kenner, N., Hyle, M., \& Vasan, N. (2004). How fast can you change your mind? The speed of topdown guidance in visual search. Vision Research, 44, 1411-1426. doi:10.1016/j.visres.2003.11.024

Woodman, G. F., \& Luck, S. J. (2007). Do the contents of visual working memory automatically influence attentional selection during visual search? Journal of Experimental Psychology: Human Perception and Performance, 33, 363-377. doi:10.1037/ 0096-1523.33.2.363 